\title{
Study of a Class of Regularizations of $1 /|x|$ using Gaussian Integrals
}

\author{
Mary Beth Ruskai * \\ Department of Mathematics \\ University of Massachusetts Lowell \\ Lowell, MA 01854 USA \\ bruskai@cs.uml.edu \\ Elisabeth Werner \\ Department of Mathematics \\ Case Western Reserve University \\ Cleveland, OH 44106 USA \\ emw2@po.cwru.edu \\ and \\ Université de Lille 1 \\ Ufr de Mathématique \\ 59655 Villeneuve d'Ascq, France \\ 16 February 1999 \\ revised 9 September 1999 and 15 November 1999
}

\begin{abstract}
This paper presents a comprehensive study of the functions $V_{m}^{p}(x)=$ $\frac{p e^{x^{p}}}{\Gamma(m+1)} \int_{x}^{\infty}\left(t^{p}-x^{p}\right)^{m} e^{-t^{p}} d t$ for $x>0, m>-1$ and $p>0$. For large $x$ these functions approximate $x^{1-p}$. The case $p=2$ is of particular importance because the functions $V_{m}^{2}(x) \approx 1 / x$ can be regarded as one-dimensional
\end{abstract}

*supported by National Science Foundation Grant DMS-97-06981 
regularizations of the Coulomb potential $1 /|x|$ which are finite at the origin for $m>-\frac{1}{2}$.

The limiting behavior and monotonicity properties of these functions are discussed in terms of their dependence on $m$ and $p$ as well as $x$. Several classes of inequalities, some of which provide tight bounds, are established. Some differential equations and recursion relations satisfied by these functions are given. The recursion relations give rise to two classes of polynomials, one of which is related to confluent hypergeometric functions. Finally, it is shown that, for integer $m$, the function $1 / V_{m}^{2}(x)$ is convex in $x$ and this implies an analogue of the triangle inequality. Some comments are made about the range of $p$ and $m$ to which this convexity result can be extended and several related questions are raised.

\section{Contents}

$\begin{array}{lll}1 & \text { Introduction } & 3\end{array}$

1.1 Definitions and background . . . . . . . . . . . . . . . . 3

1.2 Properties of $V_{m}(x)$. . . . . . . . . . . . . . . . . . 6

1.3 Convexity Summary . . . . . . . . . . . . . . . . . . . . . . . . . 9

$\begin{array}{lll}2 & \text { General } p & 10\end{array}$

$\begin{array}{lll}3 & \text { Recursion Relations and their Consequences } & 15\end{array}$

3.1 Recursion relations for $V_{m}^{p}$. . . . . . . . . . . . . . . . . . . . 15

3.2 Averaged potentials . . . . . . . . . . . . . . . . . . . 16

3.3 Polynomials defined by recursion . . . . . . . . . . . . . . . . . . 17

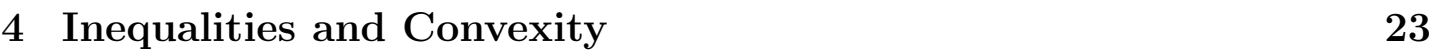

4.1 Inequalities for $V_{0}(x) \ldots \ldots \ldots \ldots$. . . . . . . . . . 23

4.2 Ratio Bounds . . . . . . . . . . . . . . . . . . . . . . . 26

$4.3 \quad$ Convexity of $1 / V_{m} \ldots \ldots \ldots \ldots$. . . . . . . . . . . . . . 27

$\begin{array}{lll}5 & \text { Proof of Ratio Bounds } & 29\end{array}$

5.1 Differential inequality . . . . . . . . . . . . . . . . . . . . . . . 29

5.2 Proof of Theorem 21 . . . . . . . . . . . . . . . . . 34

5.3 Optimality of bounds . . . . . . . . . . . . . . . . . . . 36

5.4 Extensions to general $p \ldots \ldots$. . . . . . . . . . . . . . 37 


\section{Introduction}

\subsection{Definitions and background}

In this paper we study the functions

$$
\begin{aligned}
V_{m}(x) & =\frac{2 e^{x^{2}}}{\Gamma(m+1)} \int_{x}^{\infty}\left(t^{2}-x^{2}\right)^{m} e^{-t^{2}} d t, \quad m>-1 \\
V_{-1}(x) & =\frac{1}{|x|}
\end{aligned}
$$

and their generalizations,

$$
\begin{aligned}
V_{m}^{p}(x) & =\frac{p e^{x^{p}}}{\Gamma(m+1)} \int_{x}^{\infty}\left(t^{p}-x^{p}\right)^{m} e^{-t^{p}} d t \\
V_{-1}^{p}(x) & =x^{1-p}
\end{aligned}
$$

for $0<p<\infty$. These functions are well-defined for $x>0$ and can be extended

to complex $m$ with $\Re(m)>-1$. For $\Re(m)>-\frac{1}{2}$ they are also well-defined for $x=0$. Using symmetry or the equivalent forms (4) and (7) below, they can be extended to even functions on $\mathbf{R}$ or $\mathbf{R} \backslash\{0\}$. However, it suffices to consider only non-negative $x$ and in this paper we restrict ourselves to that. We also restrict ourselves to real $m$.

Letting $p=2$ in (2) yields (国). However, because this case is more important in applications we often drop the superscript and and simply write $V_{m}(x)$ for $V_{m}^{2}(x)$.

Our interest was motivated by studies of atoms in magnetic fields where these functions arise naturally for integer $m$. $V_{m}$ can be regarded as a (twodimensional) expectation of the (three-dimensional) Coulomb potential $1 /|\mathbf{r}|$ with the state $\gamma_{m}(r, \theta)=\frac{1}{\sqrt{\pi m !}} e^{-i m \theta} r^{m} e^{-r^{2} / 2}$ (where we have used cylindrical coordinates $\mathbf{r}=(x, r, \theta)$ with the non-standard convention $r=\sqrt{y^{2}+z^{2}}$ if $\mathbf{r}=(x, y, z)$ in rectangular coordinates). The state $\gamma_{m}$ describes an electron in the lowest of the so-called "Landau levels" with angular momentum $m$ in the direction of the field. In this context, it is natural to rewrite (1) in the form

$$
\begin{aligned}
V_{m}(x) & =\frac{2}{\Gamma(m+1)} \int_{0}^{\infty} \frac{r^{2 m} e^{-r^{2}}}{\sqrt{x^{2}+r^{2}}} r d r \\
& =\frac{1}{\Gamma(m+1)} \int_{0}^{\infty} \frac{u^{m} e^{-u}}{\sqrt{x^{2}+u}} d u
\end{aligned}
$$


for $m>-1$. In this form, it is easy to see that $V_{m}(x) \approx 1 /|x|$ for large $x$. The importance of $V_{m}$ goes back at least to Schiff and Snyder 19 and played an essential role in the Avron, Herbst and Simon [2] study of the energy asymptotics of hydrogen in a strong magnetic field. More recently work in astrophysics and the work of Lieb, Solovej and Yngvason [13] on asymptotics of many-electron atoms in strong magnetic fields has renewed interest in this subject. Motivated by the LSY work, Brummelhuis and Ruskai [7, 8] have developed one-dimensional models of many-electron atoms in strong magnetic fields using the functions $V_{m}(x)$ as one-dimensional analogues of the Coulomb potential.

In the case of many-electron atoms, the anti-symmetry required by the Pauli exclusion principle suggests replacing the simple "one-electron" expectation above by an N-electron analogue in which the state $\gamma_{m}$ is replaced by a Slater determinant of such states. This is discussed in detail in [8] where it is shown that, in the simple case corresponding to $m=0 \ldots N-1$, the analogous one-dimensional potentials have the form

$$
V_{\mathrm{av}}^{N}(x)=\frac{1}{N} \sum_{m=0}^{N-1} V_{m}(x) .
$$

In Section 3 we obtain recursion relations for $V_{m}$ which, in addition to being of considerable interest in their own right, are extremely useful for studying potentials of the form (5).

For $m=0$ the function

$$
\frac{1}{\sqrt{2}} V_{0}\left(\frac{x}{\sqrt{2}}\right)=e^{x^{2} / 2} \int_{x}^{\infty} e^{-t^{2} / 2} d t
$$

occurs in many other contexts and is sometimes called the "Mills ratio" [14]. Although it has been extensively studied, the class of inequalities we consider in Section 4.1 appears to be new (although some of our bounds coincide with known inequalities in other classes) and the realization that $1 / V_{0}(x)$ is convex seems to be relatively recent [17, 18, [].

The replacement of $x^{2}$ by $x^{p}$ in (6) has been considered by Gautschi [10] and Mascioni [12], who (after seeing the preprint [15]) extended the results of Section 4.1 to this situation.

For the analysis of this generalization, it is useful to observe that (2) can be rewritten as

$$
V_{m}^{p}(x)=\frac{1}{\Gamma(m+1)} \int_{0}^{\infty} \frac{u^{m} e^{-u}}{\left(x^{p}+u\right)^{\frac{p-1}{p}}} d u
$$


In this form, it is easy to verify that $V_{m}^{p}(x) \approx x^{p-1}$ for large $x$ and that for $p=1$, $V_{m}^{1}(x)=1$ for all $m$.

Our first result shows that $V_{m}^{p}(x)$ is continuous in $m$ and that our definition for $m=-1$ is natural.

Proposition 1 For all $x>0, \lim _{m \rightarrow-1^{+}} x^{p-1} V_{m}^{p}(x)=1$.

Proof: Note that $\frac{x^{p-1}}{\left(x^{p}+u\right)^{\frac{p-1}{p}}}=\frac{1}{\left(1+\frac{u}{x^{p}}\right)^{\frac{p-1}{p}}} \quad$ so that (7) implies

$$
1-x^{p-1} V_{m}^{p}(x)=\frac{1}{\Gamma(m+1)} \int_{0}^{\infty} u^{m} e^{-u}\left[1-\left(1+\frac{u}{x^{p}}\right)^{\frac{1-p}{p}}\right] d u .
$$

Since $\Gamma(m+1)$ becomes infinite as $m \rightarrow-1$, the desired result follows if the integral on the right above remains finite. To see that this is true, it is convenient to let $g(z)=\frac{1}{z}\left(1-(1+z)^{\frac{1-p}{p}}\right)$ and note that (8) implies

$$
\left|1-x^{p-1} V_{m}^{p}(x)\right| \leq \frac{1}{\Gamma(m+1)} \int_{0}^{\infty} \frac{u^{m+1} e^{-u}}{x^{p}}\left|g\left(\frac{u}{x^{p}}\right)\right| d u
$$

It is easy to see that the large $u$ portion of this integral causes no problems since $|g(z)|$ is bounded by a polynomial in $z$ when $z>1$. (For $p \geq 1$, it is bounded uniformly by 1 ; for $0<p<1$, it is bounded by a polynomial, namely $|g(z)| \leq 1+(1+z)^{k}$, where $k \in \mathbf{N}, k \geq \frac{1}{p}$.) To see that it is also well-behaved for small $u$, we first note that for $p>0,(1+z)^{\frac{1-p}{p}}$ is analytic for $\Re(z)>-1$. Then $g(z)$ has a removable singularity at $z=0$ and can be extended to an analytic function on $\Re(z)>-1$. Thus, for small $u$, the integrand behaves like $x^{-p} u^{m+1} e^{-u}$ which ensures that the integral in (9) is finite for $m=-1$. QED

The rest of this paper is organized as follows. In the next part of this section we summarize the properties of $V_{m}$ in the important case $p=2$. We then conclude the Introduction with a summary of convexity results, including some open questions. In Section 2 we state and prove the basic properties of $V_{m}^{p}$ for general $p$. In Section 3 we derive recursion relations for $V_{m}^{p}$ and study their consequences. Among these is a connection with confluent hypergeometric functions. In Section 4.1 we prove some optimal bounds for $V_{0}$. The optimal upper bound had been established earlier independently by Wirth [18] and by Szarek and Werner [17] who also showed that the upper bound is equivalent to the convexity of $1 / V_{0}$. In Section 
4.2 we discuss several classes of inequalities, beginning with optimal bounds on $V_{0}(x)$. We then consider optimal bounds on the ratio $R_{m}(x)=V_{m}(x) / V_{m-1}(x)$ and show that these have important consequences. In particular, we show that the upper bound is equivalent to the convexity (in $x$ ) of $1 / V_{m}(x)$ and that the ratios increase with $x$. Proofs of the ratio bounds are then given in Section 5 where we also consider extensions to other $p$. Because the proof of the ratio bounds is via induction on $m$, the results of Sections 6 and 7 are only established for integer $m$. However, we believe that they hold for all $m>-1$.

\subsection{Properties of $V_{m}(x)$}

We now summarize some properties of $V_{m}(x)$ along with comments about the history and brief remarks about the proofs. Unless otherwise stated, these properties hold for $m>-1$ and $x>0$.

a) $\frac{1}{\sqrt{x^{2}+m}}>V_{m}(x)>\frac{1}{\sqrt{x^{2}+m+1}}$

where the first inequality holds for $m>0$ and the second for $m>-1$.

To prove the upper bound, which appears to be new, observe that $\mu=$ $\left[u^{m-1} e^{-u} / \Gamma(m)\right] d u$ is a probability measure on $(0, \infty)$. For fixed $x$, one can then apply Jensen's inequality to the concave function $f_{x}(u)=u\left(u+x^{2}\right)^{-1 / 2}$ to obtain

$$
\begin{gathered}
V_{m}(x)=\frac{1}{m} \int_{0}^{\infty} f_{x}(u) d \mu(u) \leq \frac{1}{m} f_{x}\left(\int_{0}^{\infty} \frac{u^{m} e^{-u} d u}{\Gamma(m)}\right) \\
=\frac{1}{m} f_{x}(m)=\frac{1}{\sqrt{x^{2}+m}} .
\end{gathered}
$$

The lower bound was proved earlier (at least for integer $m$ ) by Avron, Herbst and Simon [2] who applied a similar argument to the probability measure $\left[u^{m} e^{-u} / \Gamma(m+1)\right] d u$ and the convex function $f_{x}(u)=\left(u+x^{2}\right)^{-1 / 2}$.

b) $V_{m}(x)$ is decreasing in $m$. In particular, $V_{m+1}(x)<V_{m}(x)<\frac{1}{x}$.

The first inequality follows easily from property (a) which implies $V_{m}(x)<\frac{1}{\sqrt{x^{2}+m}}<V_{m-1}(x)$. Alternatively, one could use integration by parts on (田). The second inequality is easily verified from the integral rep-

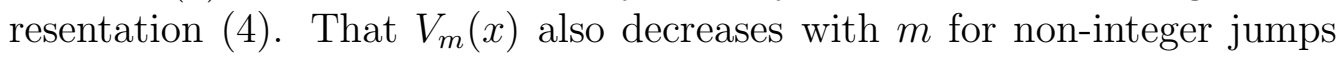
is more difficult, and the proof is postponed to Section 2 where it follows from the more general Theorem 6 . 
c) The expression $m V_{m}(x)$ is increasing in $m>-1, m \in \mathbf{R}$.

For integer jumps this holds for $m \geq-1$. Indeed, it is obvious that $-V_{-1}<$ $0 \cdot V_{0}<V_{1}$. For integer jumps with $m \geq 1$, one can use property (a) to see that

$$
m V_{m}(x)>\frac{m}{\sqrt{x^{2}+m+1}}>\frac{m-1}{\sqrt{x^{2}+m-1}}>(m-1) V_{m-1}(x) .
$$

The proof for general $m$ is postponed to Theorem 6 in Section 2. The fact that $V_{m}(x)$ is decreasing in $m$, while $m V_{m}(x)$ is increasing gives an indication of the delicate behavior of $V_{m}$.

d) For $m>-1 / 2$, the definition of $V_{m}(x)$ can be extended to $x=0$ and

$$
V_{m}(0)=\frac{\Gamma\left(m+\frac{1}{2}\right)}{\Gamma(m+1)}
$$

For integer $m$, this becomes

$$
V_{m}(0)=\frac{(2 m) !}{2^{2 m}(m !)^{2}} \sqrt{\pi}=\frac{1 \cdot 3 \cdot 5 \ldots(2 m-1)}{2 \cdot 4 \cdot 6 \ldots(2 m)} \sqrt{\pi}
$$

while for large $m$ Stirling's formula implies

$$
V_{m}(0) \approx\left(\frac{m-\frac{1}{2}}{m}\right)^{m}\left(\frac{e}{m}\right)^{1 / 2} \approx \frac{1}{\sqrt{m}}
$$

which is consistent with property (a). Boyd [6, 14] has proved the more precise estimates

$$
\frac{\sqrt{m+\frac{3}{4}+\frac{1}{32 m+48}}}{m+\frac{1}{2}}<V_{m}(0)<\frac{1}{\sqrt{m+\frac{1}{4}+\frac{1}{32 m+32}}}
$$

e) For all $m \geq 0, V_{m}$ satisfies the differential equation

$$
V_{m}^{\prime}(x)=2 x\left(V_{m}-V_{m-1}\right) .
$$

This can easily be verified using integration by parts in (4).

f) For each fixed $m \geq 0, V_{m}(x)$ is decreasing in $x$.

This follows directly from (b) and (e). 
g) For $a>0$, the expression $a V_{m}(a x)$ increases with $a$. Hence $a V_{m}(a x)>V(x)$ when $a>1$ and $a V_{m}(a x)<V(x)$ when $a<1$.

This property follows easily from the definition (3) or (4) and the observation that $\frac{a}{\sqrt{a^{2} x^{2}+u}}=\frac{1}{\sqrt{x^{2}+\frac{u}{a^{2}}}}$ is increasing in $a$. It is used in the proof of Theorem 6 and is important in the study of one-dimensional models for atoms in magnetic fields in which the electron-electron interaction takes the form of convex combinations of $\frac{1}{\sqrt{2}} V_{m}\left(\frac{\left|x_{j}-x_{k}\right|}{\sqrt{2}}\right)$.

h) $V_{0}(x)$ is convex in $x>0$; however, $V_{m}(x)$ is not convex when $m>\frac{1}{2}$.

For $m=0$, the differential equation (15) becomes $V_{0}^{\prime}(x)=2\left[x V_{0}-1\right]$. Since $x V_{0}=\int_{0}^{\infty} \frac{e^{-u}}{\sqrt{1+u / x^{2}}} d u$ is increasing for $x>0$, it follows that $V_{0}(x)$ is convex. When $m>\frac{1}{2}$ it follows from (15) and (b) that $\lim _{x \rightarrow 0} V_{m}^{\prime}(x)=0$. Since $V_{m}^{\prime}$ is negative, $V_{m}^{\prime}$ must decrease on some small interval $\left(0, x_{0}\right)$. One can also show $\lim _{x \rightarrow \infty} V_{m}^{\prime}(x)=0$, so that one expects that there is an $x_{1}$ such that $V_{m}$ is concave on $\left(0, x_{1}\right)$ and convex on $\left(x_{1}, \infty\right)$. In Section 3 we will see that the convexity is recovered for the averaged potential $V_{m}^{\text {av }}$.

i) For integer $m, 1 / V_{m}(x)$ is convex in $x>0$.

This will be proved in Section 4.2 as Theorem 23 . For large $x, 1 / V_{m}(x) \approx x$ so that the deviation from linearity is very small and the second derivative close to zero. This makes the proof quite delicate and lengthy.

The convexity of $1 / V_{m}(x)$ can be rewritten as

$$
\frac{1}{\frac{1}{2} V_{m}\left(\frac{x+y}{2}\right)} \leq \frac{1}{V_{m}(x)}+\frac{1}{V_{m}(y)} .
$$

Using property (g) with $a=\frac{1}{2}$, one easily finds that the convexity of $1 / V_{m}(x)$ implies

$$
\frac{1}{V_{m}(x+y)} \leq \frac{1}{V_{m}(x)}+\frac{1}{V_{m}(y)}
$$

This subadditivity inequality plays the role of the triangle inequality in applications. (See, e.g. [7].)

j) Asymptotic estimates:

For large $x$, it follows from property (a) that

$$
\frac{m}{2\left(x^{2}+m\right)^{3 / 2}} \leq \frac{1}{x}-V_{m}(x)<\frac{m+1}{2 x^{3}}
$$


The asymptotic expansion

$$
V_{m}(x)=\frac{1}{x}-\frac{m+1}{2 x^{3}}+\frac{3(m+2)(m+1)}{8 x^{5}}+O\left(\frac{1}{x^{7}}\right)
$$

can easily be obtained from (ब1). For details let $p=2$ in the proof of Proposition 07 which gives a similar expansion for $p>1$.

It follows from properties (a) and (c) that $V_{m}(x)$ decreases monotonically to zero for each fixed $x$ as $m \rightarrow \infty$. In fact, since $V_{m}(x)$ is decreasing in $x$ for all $m$, it suffices to show this for $x=0$ which is easy since $V_{m}(0)<m^{-1 / 2}$.

k) The Fourier transform is given by

$$
\widehat{V}_{m}(\xi) \equiv \frac{1}{\sqrt{2 \pi}} \int_{-\infty}^{\infty} V_{m}(x) e^{-i x \xi} d x=\frac{4^{m+1}}{\sqrt{2 \pi}} \int_{0}^{\infty} \frac{s^{m} e^{-s}}{\left(|\xi|^{2}+4 s\right)^{m+1}} d s
$$

This follows from (3) and the standard formula (e.g., see (v) on p. 131 of [16]) $\mathcal{F}\left(\frac{1}{\sqrt{|x|^{2}+|w|^{2}}}\right)(\xi)=\frac{1}{\sqrt{2 \pi}} \int_{0}^{\infty} \frac{1}{s} e^{-\frac{1}{2}\left(s+|w|^{2}|\xi|^{2} / s\right)} d s$ after a change in the order of integration.

\subsection{Convexity Summary}

For large $x, V_{m}^{p}(x) \approx x^{1-p}$ which is convex in $x$ for $p>1$ and concave for $p<1$. For $m>-\frac{1}{2}$ these convexity properties can not be extended to $V_{m}^{p}(x)$ on all of $(0, \infty)$; they would be inconsistent with the differential equation and monotonicity properties in Proposition 5 . However, as discussed after Proposition 11, the averaged potentials $V_{\mathrm{av}}^{p, N}$ have the same convexity as $x^{1-p}$ on the half-line.

The convexity of $1 / V_{m}^{p}(x)$ is the motivation for Sections 1 and 5 . This question is already delicate for $p=2$ and its verification becomes increasingly difficult for larger $p$. Although, as discussed in Section 5.4, we have evidence that convexity holds for all $p \geq 2$, our methods give this result only for a limited range of $p$. Moreover, because our proof is inductive, we have established convexity and ratio bounds of Section 4.2 only for integer $m$. It would be interesting to find another approach which would extend these results to non-integer $m$ and all $p \geq 2$. Since $1 / V_{m}^{p}(x) \approx x^{p-1}$ which is concave for $1<p<2$, we can not expect convexity of $1 / V_{m}^{p}$ in this range.

As discussed above, one important consequence of the convexity of $1 / V_{m}(x)$ is an analogue of the triangle inequality. For all values of $p$ we have $\left[V_{m}^{p}(x)\right]^{\frac{1}{1-p}} \approx x$ 
for large $x$ which suggests a triangle inequality of the form

$$
\left[V_{m}^{p}(x+y)\right]^{\frac{1}{1-p}} \leq\left[V_{m}^{p}(x)\right]^{\frac{1}{1-p}}+\left[V_{m}^{p}(y)\right]^{\frac{1}{1-p}} .
$$

It would be interesting to know the range of $p$ (and $m$ ) for which this holds. For $p>2$ the convexity of $1 / V_{m}^{p}(x)$ implies only the weaker inequality

$$
\left[V_{m}^{p}(x+y)\right]^{\frac{1}{1-p}} \leq 2^{\frac{p-2}{p-1}}\left(\left[V_{m}^{p}(x)\right]^{\frac{1}{1-p}}+\left[V_{m}^{p}(y)\right]^{\frac{1}{1-p}}\right)
$$

Finally, one could also ask if $V_{m}(x)$ is convex in $m$. In particular, is $2 V_{m}(x) \leq$ $V_{m+1}(x)+V_{m-1}(x)$ or, equivalently by (15), is $V_{m}^{\prime}(x)$ increasing in $m$ ?

\section{General $p$}

We now study the basic properties of $V_{m}^{p}$ in detail. As one would expect from $V_{m}^{p}(x) \approx x^{1-p}$, the behavior of $V_{m}^{p}$ is often quite different for $p>1$ and $p<$ 1. At the boundary, $p=1, V_{m}^{1}(x)=1$ for all $x$. Proposition 2 describes the monotonicity and limiting behavior of $V_{m}^{p}(x)$ as $p$ varies with $m$ and $x$ fixed. Proposition 3 gives a simple expression for $V_{m}^{p}$ in the special case that $1 / p$ is an integer.

The next four results generalize properties of Section 1.2 to general $p$. Proposition 4 generalizes the inequalities from property (a); Proposition 5 generalizes properties (d), (e), (f), and (g); and, Theorem 6 extends the monotonicity properties (b) and (c). Moreover, the proof of monotonicity for non-integer jumps is provided here. Finally, Proposition $\square$ gives the asymptotic behavior of $V_{m}^{p}(x)$ for large $x$ when $p>1$.

Proposition 2 Let $m>-1$ and $x>0$ be fixed. Then

(i) $\lim _{p \rightarrow 0} V_{m}^{p}(x)=\infty$.

(ii) For all $x \geq 1, V_{m}^{p}$ is decreasing in $p$. Moreover,

$$
\begin{aligned}
& \text { if } x>1, \quad \lim _{p \rightarrow \infty} V_{m}^{p}(x)=0, \quad \text { and } \\
& \text { if } x=1, \quad \lim _{p \rightarrow \infty} V_{m}^{p}(1)=\frac{1}{\Gamma(m+1)} \int_{0}^{\infty} \frac{u^{m} e^{-u} d u}{1+u} .
\end{aligned}
$$

(iii) For all $0<x<1$ and $m>0, \lim _{p \rightarrow \infty} V_{m}^{p}(x)=\frac{1}{m}$. 
Proof: We use the expression (đ) for $V_{m}^{p}$.

(i) Since $\lim _{p \rightarrow 0}\left(x^{p}+u\right)^{1 / p}=\infty$ for $x>1$,

$$
\lim _{p \rightarrow 0} V_{m}^{p}(x) \geq \lim _{p \rightarrow 0} \frac{1}{\Gamma(m+1)} \int_{1}^{3} \frac{u^{m} e^{-u}\left(x^{p}+u\right)^{\frac{1}{p}} d u}{x^{p}+u}=\infty .
$$

(ii) Differentiating (7) yields

$$
\begin{aligned}
\frac{d}{d p} V_{m}^{p}(x)=\frac{1}{\Gamma(m+1)} \int_{0}^{\infty} \frac{u^{m} e^{-u}}{p^{2}\left(x^{p}+u\right)^{\frac{2 p-1}{p}}} \times \\
\quad\left[(1-p) x^{p} \ln \left(x^{p}\right)-\left(x^{p}+u\right) \ln \left(x^{p}+u\right)\right] d u .
\end{aligned}
$$

For $x=1$ or $p=1$, the first term in square brackets above is zero leaving a quantity which is clearly negative. When both $x>1$ and $p>1$ both terms in (19) are clearly negative. When $x>1$ and $0<p<1$, the quantity in square brackets in (19) is negative since

$$
\begin{aligned}
& (1-p) x^{p} \ln \left(x^{p}\right)-\left(x^{p}+u\right) \ln \left(x^{p}+u\right) \\
& \leq x^{p} \ln \left(x^{p}\right)-\left(x^{p}+u\right) \ln \left(x^{p}+u\right)<0 .
\end{aligned}
$$

The last inequality follows from the fact that the function $f(w)=w \ln w$ is increasing for $w>1$. Thus, $\frac{d}{d p} V_{m}^{p}(x) \leq 0$ for all $x \geq 1$ and for all $p \in(0, \infty)$.

(iii) Since $\lim _{p \rightarrow \infty}\left(x^{p}+u\right)^{1 / p}=1$ for $x<1$,

$$
\lim _{p \rightarrow \infty} V_{m}^{p}(x)=\frac{1}{\Gamma(m+1)} \int_{0}^{\infty} u^{m-1} e^{-u} d u=\frac{1}{m} \text {. QED }
$$

Remark: The behavior for $x<1$ depends upon $m$. For "small" $m$, there is a $p_{0}$ such that $V_{m}^{p}$ decreases (below 1$)$ on $\left(0, p_{0}\right)$ and increases on $\left(p_{0}, \infty\right)$ to $\frac{1}{m}$. For "big" $m, V_{m}^{p}$ simply decreases to $\frac{1}{m}$ as $p$ increases.

The next result shows that in the special case that $1 / p$ is an integer $V_{m}^{p}$ reduces to a polynomial in $x^{p}=x^{1 / n}$ of degree $n-1$.

Proposition 3 For $n \in \mathbf{N}, n \geq 2$,

$$
V_{m}^{1 / n}(x)=\frac{1}{\Gamma(m+1)} \sum_{k=0}^{n-1}\left(\begin{array}{c}
n-1 \\
k
\end{array}\right) \Gamma(m+n-k) x^{k / n}
$$

for all $x \geq 0$ and $m>-1$. 
Proof: It follows from (7) that for $p=\frac{1}{n}$

$$
V_{m}^{1 / n}(x)=\frac{1}{\Gamma(m+1)} \int_{0}^{\infty}\left(x^{1 / n}+u\right)^{n-1} u^{m} e^{-u} d u .
$$

When $n$ is an integer $\geq 2$, the result then follows easily from the binomial expansion applied to $\left(x^{\frac{1}{n}}+u\right)^{n-1}$ and the definition of the $\Gamma$-function.

Proposition 4 For all $x>0$

$$
\frac{1}{\left(x^{p}+m+1\right)^{\frac{p-1}{p}}} \leq V_{m}^{p}(x) \leq \frac{1}{\left(x^{p}+m\right)^{\frac{p-1}{p}}} \quad \text { for } p>1,
$$

where the first inequality holds for $m>-1$ and the second for $m \geq 0$.

$$
\left(x^{p}+m+1\right)^{\frac{1-p}{p}} \geq V_{m}^{p}(x) \geq\left(x^{p}+m\right)^{\frac{1-p}{p}} \quad \text { for } \frac{1}{2} \leq p<1
$$

where the first inequality holds for $m>-1$ and the second for $m \geq 0$.

$$
V_{m}^{p}(x) \geq\left(x^{p}+m+1\right)^{\frac{1-p}{p}} \text { for } 0<p \leq \frac{1}{2},
$$

and the inequality holds for $m>-1$.

Proof: The proofs are done using Jensen's inequality as in property (a) of Section 1.2 .

Proposition 5 For all $x>0$

(i) For $m>-\frac{1}{p}, V_{m}^{p}(0)$ is defined and $V_{m}^{p}(0)=\frac{\Gamma\left(m+\frac{1}{p}\right)}{\Gamma(m+1)}$.

(ii) For all $m \geq 0, x>0, V_{m}^{p}$ satisfies the differential equation

$$
\frac{d}{d x} V_{m}^{p}(x)=p x^{p-1}\left(V_{m}^{p}(x)-V_{m-1}^{p}(x)\right) .
$$

(iii) For all $m>-1, V_{m}^{p}$ is decreasing in $x$, if $p>1$, identically equal to 1 for all $x$, if $p=1$, increasing in $x$, if $p<1$.

(iv) Let $m>-1$ and $x>0$. For $a>0$, the expression $a^{p-1} V_{m}^{p}(a x)$ increases in $a$, if $p>1$ and decreases in $a$ if $p<1$. 
Proof: The proofs are straightforward extensions of those given in Section 1.2 . In (iii), one can verify that $V_{m}^{p}$ is also increasing for $0<p<\frac{1}{2}$ by computing the derivative directly.

Theorem 6 For each fixed $x>0$, and for $m$ in the region $m>-1$,

(i) $V_{m}^{p}(x)$ is strictly decreasing in $m$ for $p>1$ and strictly increasing in $m$ for $p<1$.

(ii) $m V_{m}^{p}(x)$ is strictly increasing in $m$ for $p>1$ and strictly decreasing in $m$ for $p<1$.

Proof: To prove (i) we differentiate (7) to get

$$
\frac{d}{d m} V_{m}^{p}(x)=\frac{1}{\Gamma(m+1)} \int_{0}^{\infty} \frac{u^{m} \ln u e^{-u}}{\left(x^{p}+u\right)^{1-\frac{1}{p}}} d u-V_{m}^{p}(x) \frac{\Gamma^{\prime}(m+1)}{\Gamma(m+1)}
$$

Using the same procedure as that used (see, e.g. [1, 11]) to obtain the standard integral representation

$$
\psi(z) \equiv \frac{\Gamma^{\prime}(z)}{\Gamma(z)}=\int_{0}^{\infty}\left[\frac{e^{-s}}{s}-\frac{1}{s(1+s)^{z}}\right] d s
$$

one finds

$$
\begin{aligned}
& \frac{1}{\Gamma(m+1)} \int_{0}^{\infty} \frac{u^{m} \ln u e^{-u}}{\left(x^{p}+u\right)^{1-\frac{1}{p}}} d u \\
& \quad=\frac{1}{\Gamma(m+1)} \int_{s=0}^{\infty} \frac{d s}{s} \int_{0}^{\infty}\left[e^{-s}-e^{-s u}\right] \frac{e^{-u} u^{m}}{\left(x^{p}+u\right)^{1-\frac{1}{p}}} d u \\
& \quad=V_{m}^{p}(x) \int_{0}^{\infty} \frac{e^{-s}}{s} d s-\frac{1}{\Gamma(m+1)} \int_{0}^{\infty} \frac{d s}{s(s+1)^{m+\frac{1}{p}}} \int_{0}^{\infty} \frac{e^{-w} w^{m} d w}{\left[x^{p}(s+1)+w\right]^{1-\frac{1}{p}}} \\
& \quad=V_{m}^{p}(x) \int_{0}^{\infty} \frac{e^{-s}}{s} d s-\int_{0}^{\infty} V_{m}^{p}\left(x(s+1)^{\frac{1}{p}}\right) \frac{d s}{s(s+1)^{m+\frac{1}{p}}},
\end{aligned}
$$

where we made the change of variable $w=(s+1) u$ to obtain $V_{m}^{p}\left(x(s+1)^{\frac{1}{p}}\right)$. Now we use Proposition 4 (iv) with $a=(s+1)^{\frac{1}{p}}>1$ to obtain

$$
\begin{aligned}
\frac{1}{\Gamma(m+1)} \int_{0}^{\infty} \frac{u^{m} \ln u e^{-u}}{\left(x^{p}+u\right)^{1-\frac{1}{p}}} d u & \leq \int_{0}^{\infty} V_{m}^{p}(x)\left(\frac{e^{-s}}{s}-\frac{1}{s(s+1)^{m+1}}\right) d s \\
& =V_{m}^{p}(x) \psi(m+1)
\end{aligned}
$$


when $p>1$. For $p<1$, Proposition 4 (iv) gives the inequality in the opposite direction. Hence inserting the result in (21) yields

$$
\frac{d}{d m} V_{m}(x) \begin{cases}<0 & \text { if } p>1 \\ >0 & \text { if } p>1\end{cases}
$$

To prove (ii) it is slightly more convenient to consider the logarithmic derivative $\frac{d}{d m} \ln \left[m V_{m}^{p}(x)\right]$ and show that it is positive for $p>1$ and negative for $p<1$. Proceeding as above, we find for $p>1$

$$
\begin{aligned}
\frac{d}{d m} \ln & {\left[m V_{m}^{p}(x)\right]=\frac{1}{m}+\frac{\frac{d}{d m}\left[V_{m}^{p}(x)\right]}{V_{m}^{p}(x)} } \\
& =\frac{1}{m}+\int_{0}^{\infty} \frac{e^{-s}}{s} d s-\frac{1}{V_{m}^{p}(x)} \int_{0}^{\infty} \frac{V_{m}^{p}\left[x(s+1)^{1 / p}\right]}{s(s+1)^{m+\frac{1}{p}}} d s-\psi(m+1) \\
& <\frac{1}{m}+\int_{0}^{\infty} \frac{e^{-s}}{s} d s-\int_{0}^{\infty} \frac{1}{s(s+1)^{m}} d s-\psi(m+1) \\
& =\frac{1}{m}+\psi(m)-\psi(m+1) \\
& =\frac{1}{m}-\int_{0}^{\infty} \frac{1}{(s+1)^{m+1}}=0
\end{aligned}
$$

where we have used (22) and the following inequality with $a=(s+1)^{1 / p}$.

$$
V_{m}^{p}(a x)\left\{\begin{array}{c}
< \\
>
\end{array}\right\} a V_{m}^{p}(x) \text { for }\left\{\begin{array}{c}
p>1 \\
p<1
\end{array}\right\} .
$$

for all $a \geq 1$. This is easily verified and implies that the inequality proved above for $\frac{d}{d m} \ln \left[m V_{m}^{p}(x)\right]$ is reversed when $p<1$. QED

The following result gives the asymptotic behavior of $V_{m}^{p}(x)$ for large $x$.

Proposition 7 For $p>1, V_{m}^{p}(x)$ has the asymptotic expansion

$$
\frac{1}{x^{p-1}}-\frac{(p-1)(m+1)}{p x^{2 p-1}}+\frac{\left(2 p^{2}-3 p+1\right)\left(m^{2}+3 m+2\right)}{2 p^{2} x^{3 p-1}}+O\left(\frac{1}{x^{4 p-1}}\right)
$$

Proof: This follows from (17) since

$$
\begin{aligned}
V_{m}^{p}(x) & =\frac{1}{\Gamma(m+1) x^{p-1}} \int_{0}^{\infty} \frac{u^{m} e^{-u}}{\left(1+\frac{u}{x^{p}}\right)^{\frac{p-1}{p}}} d u \\
& =\frac{1}{\Gamma(m+1) x^{p-1}} \int_{0}^{\infty} u^{m} e^{-u}\left[1-\frac{(p-1) u}{p x^{p}}+\frac{(p-1)(2 p-1) u^{2}}{2 p^{2} x^{2 p}}+\ldots\right] d u \\
& =\frac{1}{x^{p-1}}\left[1-\frac{(p-1) \Gamma(m+2)}{p \Gamma(m+1) x^{p}}+\frac{\left(2 p^{2}-3 p+1\right) \Gamma(m+3)}{\left(2 p^{2}\right) \Gamma(m+1) x^{2 p}}+O\left(\frac{1}{x^{3 p}}\right)\right]
\end{aligned}
$$




\section{Recursion Relations and their Consequences}

\subsection{Recursion relations for $V_{m}^{p}$}

Although the case $p=2$ is of primary interest in applications, we continue to study general $p$ is this section, as the proofs for general $p$ are identical to those for $p=2$. In these recursions, our convention that $V_{-1}^{p}(x)=x^{1-p}$ plays an important role.

Proposition 8 For all $m \in \mathbf{R}, m \geq 1$, for all $x>0$,

$$
V_{m}^{p}(x)=\frac{1}{m}\left[\left(m-1+\frac{1}{p}-x^{p}\right) V_{m-1}^{p}(x)+x^{p} V_{m-2}^{p}(x)\right] .
$$

Proof: For $m=1$, one gets that

$$
V_{1}^{p}(x)=p e^{x^{p}}\left[\left(\frac{1}{p}-x^{p}\right) \int_{x}^{\infty} e^{-t^{p}} d t+x\right]=\left(\frac{1}{p}-x^{p}\right) V_{0}^{p}(x)+x^{p-1} V_{-1}^{p}(x) .
$$

For $m>1$, using (2) and integration by parts, we find

$$
\begin{aligned}
V_{m}^{p}(x)= & \frac{p e^{x^{p}}}{\Gamma(m+1)} \int_{x}^{\infty}\left(t^{p}-x^{p}\right)^{m-1}\left(t^{p}-x^{p}\right) e^{-t^{p}} d t \\
= & \frac{p e^{x^{p}}}{m \Gamma(m)}\left[\left(-x^{p} \int_{x}^{\infty} e^{-t^{p}}\left(t^{p}-x^{p}\right)^{m-1} d t\right.\right. \\
& \left.\quad+\frac{1}{p} \int_{x}^{\infty} e^{-t^{p}}\left(\left(t^{p}-x^{p}\right)^{m-1}+(m-1) p t^{p}\left(t^{p}-x^{p}\right)^{m-2} t^{2}\right) d t\right] \\
= & \frac{p e^{x^{p}}}{m \Gamma(m)}\left[\left(m-1+\frac{1}{p}-x^{p}\right) \int_{x}^{\infty} e^{-t^{p}}\left(t^{p}-x^{p}\right)^{m-1} d t\right. \\
& \left.\quad+(m-1) x^{p} \int_{x}^{\infty}\left(t^{p}-x^{p}\right)^{m-2} e^{-t^{2}} d t\right] \\
= & \frac{1}{m}\left[\left(m-1+\frac{1}{p}-x^{p}\right) V_{m-1}^{p}(x)++x^{p} V_{m-2}^{p}(x)\right]
\end{aligned}
$$

Repeated application of (24) gives a useful corollary. For $m \in \mathbf{R}$, let $\lfloor m\rfloor$ denote the "floor" of $m$, i.e., the largest natural number less than or equal to $m$.

Corollary $\mathbf{9}$ Let $m \in \mathbf{R}, m \geq 1$ and let $n \in \mathbf{N}$ such that $n \leq\lfloor m\rfloor$. Then

$$
\begin{aligned}
V_{m}^{p}(x)=\frac{1}{p m}\left[\left(1-p x^{p}\right) V_{m-1}^{p}(x)+V_{m-2}^{p}(x)+\ldots\right. \\
\left.\quad+\ldots[p(m-n)+1] V_{m-n}^{p}(x)+p x^{p} V_{m-n-1}^{p}(x)\right] .
\end{aligned}
$$


In particular, if $m$ is a positive integer, then

$$
V_{m}^{p}(x)=\frac{1}{p m}\left[\left(1-p x^{p}\right) V_{m-1}^{p}(x)+\sum_{k=0}^{m-2} V_{k}^{p}(x)+p x^{p} V_{-1}^{p}(x)\right] .
$$

The expression (26) is well-defined for $x=0$. Putting $x=0$ and using Proposition 5 (i), we obtain the (presumably well-known) identity

$$
\frac{\Gamma\left(m+\frac{1}{p}\right)}{\Gamma(m+1)}=\frac{1}{p m} \sum_{k=0}^{m-1} \frac{\Gamma\left(k+\frac{1}{p}\right)}{\Gamma(k+1)}
$$

\subsection{Averaged potentials}

These recursion relations are quite useful for studying the average of the first $N$ of the $V_{m}$. For $N$ a positive integer, we extend (5) to

$$
V_{\mathrm{av}}^{p, N}(x)=\frac{1}{N} \sum_{m=0}^{N-1} V_{m}^{p}(x)
$$

Note that for $p=1, V_{\mathrm{av}}^{1, N}(x)=1$, for all $x \geq 0$.

The next result follows immediately from (26).

Corollary $10 \quad V_{\mathrm{av}}^{p, N}(x)=p V_{N}^{p}(x)-\frac{p x^{p}}{N}\left[V_{-1}^{p}(x)-V_{N-1}^{p}(x)\right]$.

For the important case $p=2$, this reduces to

$$
V_{\mathrm{av}}^{N}(x)=2 V_{N}(x)-\frac{2 x^{2}}{N}\left[V_{-1}(x)-V_{N-1}(x)\right]
$$

The function $V_{0}(|x|)$ is convex on $(0, \infty)$ but has a cusp at $x=0$. However, as discussed in property (f), for higher $m$ both the convexity and cusp are lost. Thus, for higher $m$, the $V_{m}$ are somewhat smoother than one might want for one-dimensional approximations to the Coulomb potential. The next result, although straightforward, is important because it implies that the averaged potentials $V_{\mathrm{av}}^{N}(x)$ retain the cusp and convexity properties of $V_{0}$ near the origin.

Proposition 11 The function $V_{\mathrm{av}}^{N}(x)$ is convex for all $x>0$ and

$$
\lim _{x \rightarrow 0+} \frac{d}{d x} V_{\mathrm{av}}^{N}(x)=-\frac{2}{N} .
$$


Proof: Using (5) and (15) one finds

$$
\frac{d}{d x} N V_{\mathrm{av}}^{N}(x)=\sum_{m=0}^{N-1} 2 x\left[V_{m}(x)-V_{m-1}\right]=2 x\left[V_{N-1}-V_{-1}\right]=2 x V_{N-1}-2 .
$$

Therefore, to show that $V_{\mathrm{av}}^{N}$ is convex, we need to show that

$$
x V_{N-1}=\frac{1}{\Gamma(N)} \int_{0}^{\infty} \frac{u^{N-1} e^{-u} d u}{\left(1+\frac{u}{x^{2}}\right)^{\frac{1}{2}}}
$$

is increasing. This holds as, for $x>0$, the function $h_{u}(x)=\left[1+u / x^{2}\right]^{-1 / 2}$ is increasing.

Similarly, one can show that for $p>1, V_{\mathrm{av}}^{p, N}$ is convex on $(0, \infty)$ and

$$
\lim _{x \rightarrow 0+} \frac{d}{d x} V_{\mathrm{av}}^{p, N}(x)=-\frac{p}{N} .
$$

For $p<1$, the derviative becomes infinite at the origin; however, concavity of $V_{\mathrm{av}}^{p, N}$ on $(0, \infty)$ still holds.

\subsection{Polynomials defined by recursion}

We now observe that by repeatedly using (24) to eliminate the $V_{m}^{p}$ with the largest value of $m$ from (25) allows us to write $V_{m}^{p}$ in terms of the two "lowest" functions (e.g., $V_{0}^{p}$ and $V_{-1}^{p}$ in the case of integer $m$ ) and that the coefficients in such expressions define two classes of polynomials related to confluent hypergeometric functions. We discuss the properties of these polynomials in some detail. First, we make the statement above explicit.

Corollary 12 For $m \geq 1$ there are polynomials $P_{m}^{p}(y)$ and $Q_{m}^{p}(y)$ of degree $\lfloor m\rfloor$ such that

$$
V_{m}^{p}(x)=P_{m}^{p}\left(x^{p}\right) V_{m-\lfloor m\rfloor}^{p}(x)+x^{p} Q_{m-1}^{p}\left(x^{p}\right) V_{m-\lfloor m\rfloor-1}^{p}(x) .
$$

In the case of integer $m(30)$ becomes

$$
\begin{aligned}
V_{m}^{p}(x) & =P_{m}^{p}\left(x^{p}\right) V_{0}^{p}(x)+x^{p} Q_{m-1}^{p}\left(x^{p}\right) V_{-1}^{p}(x) \\
& =P_{m}^{p}\left(x^{p}\right) V_{0}^{p}(x)+x Q_{m-1}^{p}\left(x^{p}\right)
\end{aligned}
$$

where the second expression follows from our convention $V_{-1}^{p}(x)=x^{1-p}$. We define $P_{m}^{p}(y)=1$ for $m \in[0,1)$ and $Q_{m}^{p}(y)=0$ for $m \in[-1,0)$. Then (30) holds trivially for $m \in[0,1)$. 
Proof: The desired polynomials are defined recursively. First let

$$
\begin{aligned}
P_{m}^{p}(y) & =\frac{1}{m}\left(m-1+\frac{1}{p}-y\right) \text { for } m \in[1,2), \quad \text { and } \\
Q_{m}^{p}(y) & =\frac{1}{m+1} \text { for } m \in[0,1) .
\end{aligned}
$$

Then (30) holds because it is equivalent to (24) for $m \in[1,2)$. For $m \geq 2$ define $P_{m}^{p}(y)$ by

$$
P_{m}^{p}(y)=\frac{1}{m}\left[\left(m-1+\frac{1}{p}-y\right) P_{m-1}^{p}(y)+y P_{m-2}^{p}(y)\right]
$$

and for $m \geq 1$ define $Q_{m}^{p}(y)$ by

$$
Q_{m}^{p}(y)=\frac{1}{m+1}\left[\left(m+\frac{1}{p}-y\right) Q_{m-1}^{p}(y)+y Q_{m-2}^{p}(y)\right] .
$$

It is straightforward to use induction to check that (24) yields (30). QED

We now restrict ourselves to $m \in \mathbf{N}$ and study these polynomials in more detail. The first few polynomials are given in the following Table.

$$
\begin{array}{ccc}
m & P_{m}^{p} & Q_{m}^{p} \\
0 & 1 & 1 \\
1 & \frac{1}{p}-y & \frac{1}{2}\left(1+\frac{1}{p}-y\right) \\
2 & \frac{1}{2}\left[\left(y-\frac{1}{p}\right)^{2}+\frac{1}{p}\right] & \frac{1}{3}\left[y+\frac{1}{2}\left(1+\frac{1}{p}-y\right)\left(2+\frac{1}{p}-y\right)\right]
\end{array}
$$

The following useful results, which hold for $m \geq 1$, are easily checked by induction. $B(x, y)=\frac{\Gamma(x) \Gamma(y)}{\Gamma(x+y)}$ is the Beta function.

$$
\begin{aligned}
P_{m}^{p}(0) & =\frac{\Gamma\left(m+\frac{1}{p}\right)}{\Gamma(m+1) \Gamma\left(\frac{1}{p}\right)}=\frac{1}{m B\left(m, \frac{1}{p}\right)} \\
Q_{m}^{p}(0) & =\frac{\Gamma\left(m+1+\frac{1}{p}\right)}{\Gamma(m+2) \Gamma\left(\frac{1}{p}\right)}=\frac{1}{(m+1) B\left(m+1, \frac{1}{p}\right)}
\end{aligned}
$$




$$
\begin{aligned}
P_{m}^{p}(y) & =\frac{1}{m}\left[\frac{1}{p} \sum_{j=0}^{m-1} P_{j}^{p}(y)-y P_{m-1}^{p}(y)\right], \quad \text { and } \\
Q_{m}^{p}(y) & \left.=\frac{1}{m+1}\left[\frac{1}{p} \sum_{j=0}^{m-1} Q_{j}^{p}(y)-y Q_{m-1}^{p}(y)+1\right]\right] .
\end{aligned}
$$

We now obtain two expressions for $\frac{d}{d x} V_{m}^{p}(x)$. First, observe that using (31) in (20) yields

$\frac{d}{d x} V_{m}^{p}(x)=p x^{p-1}\left(\left[P_{m}^{p}\left(x^{p}\right)-P_{m-1}^{p}\left(x^{p}\right)\right] V_{0}^{p}(x)+x\left[Q_{m-1}^{p}\left(x^{p}\right)-Q_{m-2}^{p}\left(x^{p}\right)\right]\right)$.

Differentiating (31) yields after some simplifications

$$
\begin{aligned}
\frac{d}{d x} V_{m}^{p}(x)=p x^{p-1}[ & \left(P_{m}^{p}\right)^{\prime}\left(x^{p}\right) V_{0}^{p}(x)+P_{m}^{p}\left(x^{p}\right)\left[V_{0}^{p}(x)-V_{-1}^{p}(x)\right] \\
& \left.+x^{p}\left(Q_{m-1}^{p}\right)^{\prime}\left(x^{p}\right) V_{-1}^{p}(x)+\frac{1}{p} Q_{m-1}^{p}\left(x^{p}\right) V_{-1}^{p}(x)\right]
\end{aligned}
$$

where $\left(P_{m}^{p}\right)^{\prime}(y)$ denotes $\frac{d}{d y} P_{m}^{p}(y)$. Equating these expressions yields

$$
\begin{aligned}
& -\left[\left(P_{m}^{p}\right)^{\prime}\left(x^{p}\right)+P_{m-1}^{p}\left(x^{p}\right)\right] V_{0}^{p}(x)= \\
& \quad\left[x^{p}\left(Q_{m-1}^{p}\right)^{\prime}\left(x^{p}\right)-P_{m}^{p}\left(x^{p}\right)+\left(\frac{1}{p}-x^{p}\right) Q_{m-1}^{p}\left(x^{p}\right)+x^{p} Q_{m-2}^{p}\left(x^{p}\right)\right] V_{-1}^{p}(x)
\end{aligned}
$$

This provides motivation for the following

Lemma 13 For $m \in \mathbf{N}, m \geq 1$,

$$
\begin{aligned}
\frac{d}{d y} P_{m}^{p}(y) & =-P_{m-1}^{p}(y), \quad \text { and } \\
y \frac{d}{d y} Q_{m-1}^{p}(y) & =P_{m}^{p}(y)-(m+1) Q_{m}^{p}(y)+m Q_{m-1}^{p}(y)
\end{aligned}
$$

Proof: We first prove (39) by induction. It can be verified for $m=1,2$ using the Table above. Then using (34), we find

$$
\begin{aligned}
m & \frac{d}{d y} P_{m}^{p}(y) \\
& =\left(m-1+\frac{1}{p}-y\right) \frac{d}{d y} P_{m-1}^{p}(y)-P_{m-1}^{p}(y)+y \frac{d}{d y} P_{m-2}^{p}(y)+P_{m-2}^{p}(y) \\
& =-P_{m-1}^{p}(y)-\left(m-2+\frac{1}{p}-y\right) P_{m-2}^{p}(y)-y P_{m-3}^{p}(y) \\
& =-m P_{m-1}^{p}(y) .
\end{aligned}
$$


This implies that the coefficient of $V_{0}^{p}$ in (38) is identically zero. Therefore the coefficient of $V_{-1}^{p}$ must also be identically zero. Substituting $y=x^{p}$ gives

$$
\begin{aligned}
y \frac{d}{d y} Q_{m-1}^{p}(y) & =P_{m}^{p}(y)+\left(y-\frac{1}{p}\right) Q_{m-1}^{p}(y)-y Q_{m-2}^{p}(y) \\
& =P_{m}^{p}(y)-(m+1) Q_{m}^{p}(y)+m Q_{m-1}^{p}(y)
\end{aligned}
$$

where we used (35). QED

Note that since the left side of (40) is a polynomial of degree $m-1$, this implies the coefficients of the $y^{m}$ terms in $P_{m}^{p}$ and $(m+1) Q_{m}^{p}(y)$ are identical. In fact, one can use (34) and (35) to see that the leading terms of $P_{m}^{p}$ is $(-1)^{m} y^{m} / m$ ! and that of $Q_{m}^{p}$ is $(-1)^{m} y^{m} /(m+1)$ !

A set of polynomials $\left\{p_{n}(x)\right\}$ belongs to the class known as Appell polynomials [4] if they satisfy $\frac{d}{d x} p_{n}(x)=p_{n-1}(x)$. Therefore, (39) implies that for each fixed $p$, the set $(-)^{m} P_{m}^{p}(y)$ forms a family of Appell polynomials.

One can use (39) in (34) to replace $P_{m-1}^{p}$ and $P_{m-2}^{p}$ by derivatives of $P_{m}^{p}$ and obtain a second order differential equation satisfied by $P_{m}^{p}$. This allows us to obtain a relationship between the polynomials $P_{m}^{p}$ and confluent hypergeometric functions, which we denote ${ }_{1} F_{1}(\alpha, \gamma, y)$.

Theorem 14 For $m \in \mathbf{N}, m \geq 1, P_{m}^{p}(y)$ satisfies the differential equation

$$
y \phi^{\prime \prime}(y)-\left(m-1+\frac{1}{p}-y\right) \phi^{\prime}(y)-m \phi(y)=0 .
$$

Standard techniques show that (41) has a polynomial solution of the form $\phi(y)=\sum_{k=0}^{m} b_{k} y^{k}$ with $b_{k}=-\frac{m+1-k}{k\left(m+\frac{1}{p}-k\right)} b_{k-1}, k \geq 1, b_{0} \neq 0$ arbitrary, and a second solution of the form $\phi(y)=\sum_{k=0}^{\infty} c_{k} y^{k+m+1 / p}$ with $c_{k}=-\frac{k-1+1 / p}{k\left(m+\frac{1}{p}+k\right)} c_{k-1}, k \geq 1$, $c_{0} \neq 0$ arbitrary. Since $P_{m}^{p}(0)=\frac{1}{m B\left(m, \frac{1}{p}\right)}$, we conclude that $b_{k}=\frac{(-1)^{k}}{k !(m-k) B\left(m-k, \frac{1}{p}\right)}$ and

$$
P_{m}^{p}(y)=\sum_{k=0}^{m} \frac{(-1)^{k} \Gamma\left(m+\frac{1}{p}-k\right)}{\Gamma(k+1) \Gamma(m+1-k) \Gamma\left(\frac{1}{p}\right)} y^{k} .
$$

The restriction that $1 / p$ be non-integer in the next result is neither serious, nor unexpected, in view of Proposition 3 .

Corollary 15 Let $p \neq \frac{1}{n}$ for $n \in \mathbf{N}$. Then

$$
P_{m}^{p}(y)=\frac{1}{m B\left(m, \frac{1}{p}\right)} e_{1}^{-y} F_{1}\left(1-\frac{1}{p}, 1-\frac{1}{p}-m, y\right)
$$


Proof: Write $\phi(y)=e^{-y} \widehat{\phi}(y)$. Then it follows from (41) that $\widehat{\phi}$ satisfies

$$
y \widehat{\phi}^{\prime \prime}(y)-\left(m-1+\frac{1}{p}+y\right) \widehat{\phi}^{\prime}(y)-\left(1-\frac{1}{p}\right) \widehat{\phi}(y)=0 .
$$

which has the form of the differential equation satisfied by the confluent hypergeometric function. Comparing the behavior of $P_{m}^{p}(y)$ near $y=0$ with that of the well-known solutions to (43) suffices to complete the proof. QED

It is well-known [3] that for real $\alpha$ and $\gamma,{ }_{1} F_{1}(\alpha, \gamma, y)$ has at most finitely many zeros on the real line. Hence, the same holds for $P_{m}^{p}$. In fact, we can show that $P_{m}^{p}$ has no zeros when $m$ is even and exactly one when $m$ is odd.

To show this, it is convenient to introduce the new variable $z=\frac{1}{p}-y$ and write $P_{m}^{p}(y)=\tilde{P}_{m}^{p}\left(\frac{1}{p}-y\right)$. The first few of these polynomials are $\tilde{P}_{0}^{p}(z)=1$, $\tilde{P}_{1}^{p}(z)=z$, and $\tilde{P}_{2}^{p}(z)=\frac{1}{2}\left(z^{2}+\frac{1}{p}\right)$.

Lemma 16 For $m \in \mathbf{N}, m \geq 2$, the polynomials $\tilde{P}_{m}^{p}(z)$ satisfy

(i) $\tilde{P}_{m}^{p}(z)=\frac{1}{m}\left[(m-1+z) \tilde{P}_{m-1}^{p}(z)+\left(\frac{1}{p}-z\right) \tilde{P}_{m-2}^{p}(z)\right]$,

(ii) $\tilde{P}_{m}^{p}(z)=\frac{1}{m}\left[\frac{1}{p} \sum_{j=0}^{m-2} \tilde{P}_{j}^{p}(z)+z \tilde{P}_{m-1}^{p}(z)\right]$, and

(iii) $\frac{d}{d z} \tilde{P}_{m}^{p}(z)=\tilde{P}_{m-1}^{p}(z)$.

Proof: Follows immediately from substitution in (34), (36) and (39).

Corollary 17 For $m \in \mathbf{N}, m \geq 0$, all coefficients in the polynomials $\tilde{P}_{m}^{p}(z)$ are positive.

Proof: This follows immediately from the explicit expressions above for $\tilde{P}_{m}^{p}$ when $m=0,1$ and part (ii) of Lemma 16 .

Proposition 18 Let $m \in \mathbf{N}, m \geq 1$

If $m$ is even, $\tilde{P}_{m}^{p}(z) \geq 0$.

If $m$ is odd, $\tilde{P}_{m}^{p}(z)$ has exactly one root $z_{m}$.

Moreover, the roots form a strictly decreasing sequence with $-m+1 \leq z_{m} \leq 0$. 
Proof: First note that Corollary 17 implies that $\tilde{P}_{m}^{p}(z) \geq 0$ for all $z \geq 0$ and $\lim _{z \rightarrow \infty} \tilde{P}_{m}^{p}(z)=\infty$. Now we claim that

$$
\lim _{z \rightarrow-\infty} \tilde{P}_{m}^{p}(z)=\left\{\begin{aligned}
-\infty & \text { if } \mathrm{m} \text { is odd } \\
\infty & \text { if } \mathrm{m} \text { is even }
\end{aligned}\right.
$$

This can easily be verified by induction using part(i) of Lemma 16 (i) above.

For $m$ odd, $\tilde{P}_{m}^{p}(z)$ has at least one root $z_{m}$. We now prove by induction that $z_{m}$ is the only root if $m$ is odd and that $\tilde{P}_{m}^{p}(z) \geq 0$ for all $z$ if $m$ is even. The induction hypothesis is easily seen to hold for $m=0,1$. Suppose it is true up to $m-1$ and consider $\tilde{P}_{m}^{p}$, with $m$ odd. Then, by Lemma 16 (iii), $\frac{d}{d z} \tilde{P}_{m}^{p}(z)=\tilde{P}_{m-1}^{p}(z)$. Since $m-1$ is even, by the induction hypothesis $\tilde{P}_{m-1}^{p}(z) \geq 0$. Thus $\tilde{P}_{m}^{p}(z)$ is increasing for all $z \in \mathbf{R}$, which implies that for $m$ odd $\tilde{P}_{m}^{p}(z)$ has only one root. Since $\tilde{P}_{m}^{p}(z)>0$ when $z>0$ that one root must satisfy $z_{m} \leq 0$.

If $m$ is even, then $\frac{d}{d z} \tilde{P}_{m}^{p}(z)=\tilde{P}_{m-1}^{p}(z)$ which, by the induction hypothesis, has exactly one root $z_{m-1} \leq 0$. Therefore $\tilde{P}_{m}^{p}(z)$ has a local extremum at $z_{m-1}$. Since $\frac{d^{2}}{(d z)^{2}} \tilde{P}_{m}^{p}(z)=\tilde{P}_{m-2}^{p}(z)$ and $m-2$ is even, $\tilde{P}_{m-2}^{p}(z) \geq 0$ by the induction hypothesis and $z_{m-1}$ is a local minimum for $\tilde{P}_{m}^{p}(z)$. By Lemma 16 (i) we have

$$
m \tilde{P}_{m}^{p}\left(z_{m-1}\right)=\left(\frac{1}{p}-z_{m-1}\right) \tilde{P}_{m-2}^{p}\left(z_{m-1}\right)
$$

since $\tilde{P}_{m-1}^{p}\left(z_{m-1}\right)=0$. But by the induction hypothesis $z_{m-1} \leq 0$ and $\tilde{P}_{m-2}^{p}\left(z_{m-1}\right)>$ 0 so that $\tilde{P}_{m}^{p}\left(z_{m-1}\right)>0$ as required.

It remains to be shown that the roots are decreasing and bounded below by $-(m-1)$. Both can be easily checked for $m=1,3$ and then proved by induction using Lemma 16 (i). We now let $m$ be odd. Since $\tilde{P}_{m}^{p}(z)$ is increasing to show that $z_{m}>-m+1$, it suffices to show that $\tilde{P}_{m}^{p}(-m+1)<0$. For $z=-m+1$ the recursion relation reduces to

$$
m \tilde{P}_{m}^{p}(-m+1)=\left(\frac{1}{p}+m-1\right) \tilde{P}_{m-2}^{p}(-m+1)
$$

which is negative by the induction assumption hypothesis that $z_{m-2} \geq-m+3$. To show that $z_{m}<z_{m-2}$ it suffices to show that $\tilde{P}_{m}^{p}\left(z_{m-2}\right)>0$. But

$$
m \tilde{P}_{m}^{p}\left(z_{m-2}\right)=\left(m-1+z_{m-2}\right) P_{m-1}^{p}\left(z_{m-2}\right) \geq 0
$$

since $P_{m-2}^{p}\left(z_{m-2}\right)=0, z_{m-2}>-m+3>-m+1$, and $P_{m-1}^{p}(z)$ is positive. QED

We now restate the results above in terms of the behavior of the original polynomials $P_{m}^{p}(y)$. 
Corollary 19 Let $m \in \mathbf{N}, m \geq 1$. Then

(i) If $m$ is even, then $P_{m}^{p}(y) \geq 0$ for all $y \in \mathbf{R}$.

(ii) If $m$ is odd, then $P_{m}^{p}(y)$ has exactly one root $y_{m} \geq 0$.

(iii) For all $m \geq 1$,

$$
\begin{aligned}
\lim _{y \rightarrow-\infty} P_{m}^{p}(y) & =\infty \\
\lim _{y \rightarrow \infty} P_{m}^{p}(y) & =\left\{\begin{aligned}
-\infty & \text { if } m \text { is odd } \\
\infty & \text { if } m \text { is even }
\end{aligned}\right.
\end{aligned}
$$

Although we were able to obtain an explicit expression for the polynomials $P_{m}^{p}(x)$ relating them to confluent hypergeometric functions and analyze their behavior in some detail, we do not much information about $Q_{m}^{p}(x)$. This is, at least in part, because (40) mixes $Q_{m}^{p}(x)$ and $P_{m}^{p}(x)$ and does not lead directly to a differential equation for $Q_{m}^{p}(x)$. It would be interesting to know more about the polynomials $Q_{m}^{p}(x)$.

\section{Inequalities and Convexity}

\subsection{Inequalities for $V_{0}(x)$}

We first illustrate our strategy by proving a special class of inequalities for $V_{0}$. The convexity of $1 / V_{0}(x)$ follows directly from the optimal upper bound in this class as given in Theorem 20 below. Although, as discussed at the end of this section, these inequalities generalize to $V_{m}$ the resulting upper bound is not sufficient to establish the convexity of $1 / V_{m}$. For this we need a bound on the ration $V_{m}(x) / V_{m-1}(x)$. Nevertheless, these simple inequalities for $V_{0}$, which can also be interpreted as ratio bounds, are of some interest in their own right in a variety of applications. Because the geometric strategy is also used in our more complex proofs of ratio bounds, we think there is some merit in presenting it first here.

We now define

$$
g_{k}(x)=\frac{k}{(k-1) x+\sqrt{x^{2}+k}} .
$$

Theorem 20 For $x \geq 0$

$$
g_{\pi}(x) \leq V_{0}(x)<g_{4}(x)
$$


and these inequalities are optimal for functions of the form (44) with equality only at $g_{\pi}(0)=V_{0}(0)=\sqrt{\pi}$.

Proof: It is easy to see that the family of functions $g_{k}(x)$ is increasing in $k$ and that $0<g_{k}(x)<1 / x$. In order to prove that the upper bound is optimal, we first observe that $g_{k}^{\prime}(x)=-k\left[g_{k}(x)\right]^{2}\left[x+(k-1) \sqrt{x^{2}+k}\right] / \sqrt{x^{2}+k}$ and $x g_{k}(x)-1=$ $-k g_{k}(x) /\left[x+\sqrt{x^{2}+k}\right]$. Then one can verify that

$$
\begin{aligned}
g_{k}^{\prime}(x)> & 2\left[x g_{k}(x)-1\right] \\
& \Longleftrightarrow \frac{k}{\sqrt{x^{2}+k}} \frac{(k-1) \sqrt{x^{2}+k}+x}{(k-1) x+\sqrt{x^{2}+k}}<\frac{2 k}{x+\sqrt{x^{2}+k}} \\
& \Longleftrightarrow(k-2) x^{2}+k(k-3)<(k-2) x \sqrt{x^{2}+k} \\
& \Longleftrightarrow x^{2}(k-2)(k-4)+k(k-3)^{2}<0
\end{aligned}
$$

when $k>3$. We now restrict attention to $3 \leq k \leq 4$ and let $h_{k}(x)=g_{k}(x)-V_{0}(x)$. For $k=4$, the expression (46) implies $g_{4}^{\prime}(x)<2\left[x g_{k}(x)-1\right]$ so that $h_{4}^{\prime}(x)<$ $2 x h_{4}(x)$ for all $x \geq 0$; whereas for $k<4$ this holds only for $x<a_{k}=\sqrt{\frac{k(k-3)^{2}}{(k-2)(4-k)}}$. Since both $V_{0}(x)$ and $g_{k}(x)$ are positive and bounded above by $1 / x$, their difference also satisfies $\left|h_{k}(x)\right|<1 / x \rightarrow 0$.

For $k=4$, if $h_{4}(x) \leq 0$ for some $x>0$, then $h_{4}^{\prime}(x)<2 x h_{4}(x)$ is negative and thus $h_{4}$ is negative and strictly decreasing from a certain $x$ on, which contradicts $\lim _{x \rightarrow \infty} h_{4}(x)=0$. Thus $h_{4}(x)>0$ so that $g_{4}(x)>V_{0}(x)$, for all $x$. Now suppose that for some $k<4, g_{k}$ is an upper bound, i.e. $h_{k}(x) \geq 0$ for all $x \geq 0$. In particular, $h_{k}(x) \geq 0$ for all $x>a_{k}$. For $k<4$, we find however that $h_{k}^{\prime}(x)>$ $2 x h_{k}(x)$ holds for $x>a_{k}$. Thus we get $h_{k}(x) \geq 0$ and strictly increasing for all $x>a_{k}$ which contradicts $\lim _{x \rightarrow \infty} h_{k}(x)=0$. Thus the upper bound can not hold when $x>a_{k}$ and $k<4$. The lower bound also fails for $k>\pi$ since then $h_{k}(0)=g_{k}(0)-V_{0}(0)=\sqrt{k}-\sqrt{\pi}>0$.

To establish the improved lower bound $g_{\pi} \leq V_{0}(x)$ we note that the argument above implies that $h_{k}(x)$ is negative for $x>a_{k}$ and $3<k \leq \pi$. However for $k<\pi$ we have $h_{k}(0)<0$ so that $h_{k}(x)$ is also negative for very small $x$. If $h_{k}(x)$ is ever non-negative, we can let $b$ denote the first place $h_{k}(x)$ touches or crosses the x-axis, i.e., $h_{k}(b)=0$ and $h_{k}(x)<0$ for $x<b$. Then $h_{k}$ must be increasing on some interval of the form $\left(x_{0}, b\right)$. However, by the remarks above, $h_{k}(b)=0$ implies $b \leq a_{k}$ so that $h_{k}^{\prime}(x)<2 x h_{k}(x)<0$ on $\left(x_{0}, b\right)$. Since this contradicts $h_{k}$ increasing on $\left(x_{0}, b\right)$, we must have $h_{k}(x)<0$ for all $x \geq 0$ if $k<\pi$.

Thus we have proved the lower bound $g_{k}(x)<V_{0}(x)$ on $[0, \infty)$ for $k<\pi$. Since $g_{k}$ is continuous and increasing in $k$, it follows that $g_{\pi}(x) \leq V_{0}(x)$. To show 
that this inequality is strict except at $x=0$, note that the right derivative of $h_{k}$ at 0 satisfies $h_{k}^{\prime}(0)=2-k$ so that $h_{\pi}^{\prime}(0)<0$ and $h_{\pi}(x)$ is negative at least on some small interval $\left(0, x_{1}\right)$. Then we can repeat the argument above to show that $h_{\pi}(x)<0$ if $x>0$. QED

As discussed in [7, 17, 18] the upper bound implies the convexity of $1 / V_{0}(x)$ on $(0, \infty)$; in fact, it is not hard to use the fact that (15) reduces to $\frac{d}{d x} V_{0}(x)=2\left(x V_{0}-\right.$ 1) to see that the upper bound is equivalent to convexity. It was established independently by Wirth [18] and by Szarek and Werner [17]. (The latter actually proved slightly more by using (1) to define an asymmetric extension of $V_{0}(x)$ to negative $x$. They showed in [17] that this extension is convex for $x>-\frac{1}{\sqrt{2}}$.)

Both bounds in (45) are sharper than the inequalities of Komatsu [9, 14]. The weaker lower bound $g_{3}(x)<V_{0}(x)$ was used in [7] to show that the function $\left[1 / V_{0}(x)-x\right]^{2} / V_{0}(x)$ is decreasing for $x \geq 0$. The lower bound $g_{\pi}(x) \leq V_{0}(x)$ was established earlier by Boyd [5] as the optimal bound in a different class of inequalities. There is an extensive literature (see. e.g. [14]) on bounds for $V_{0}(x)$; however, the class of inequalities obtained using functions of the form $g_{k}(x)$ does not seem to have been considered before so that the optimality of bounds of this type for $k=\pi$ and $k=4$ seems new.

Mascioni [12] generalized the upper bound to $p \geq 2$ for which he showed

$$
V_{0}^{p}(x)<\frac{4 p}{3 p x^{p-1}+\sqrt{p^{2} x^{2 p-2}+8 p(p-1) x^{p-2}}}
$$

and also showed that this implies convexity of $1 / V_{0}^{p}(x)$ for $p \geq 2$.

In view of Property (a) of Section 1.2, it would seem natural to try to generalize (20) using functions of the form

$$
g_{k}^{m}(x)=\frac{k}{(k-1) x+\sqrt{x^{2}+m+k}} .
$$

Note that the functions $g_{k}^{m}$ are increasing in $k$ and that $\lim _{k \rightarrow \infty} g_{k}^{m}(x)=\frac{1}{x}$. Therefore Property (a) implies that

$$
g_{1}^{m}(x) \leq V_{m}(x)<\lim _{k \rightarrow \infty} g_{k}^{m}(x) .
$$

As $g_{k}^{m}$ is continuous in $k$, there must exist $i_{m}$ and $j_{m}$ such that

$$
g_{i_{m}}^{m}(x) \leq V_{m}(x)<g_{j_{m}}^{m}(x)
$$


However, we have not obtained explicit expressions for $i_{m}$ and $j_{m}$. One might expect that the optimal lower bound occurs when $i_{m}$ is chosen to satisfy $g_{i_{m}}^{m}(0)=$ $V_{m}(0)$. However, numerical evidence shows that this is false; in fact, this choice for $i_{m}$ does not even yield an inequality.

\subsection{Ratio Bounds}

One of our main goals is to show that the function $\frac{1}{V_{m}(x)}$ is convex for integer $m \geq 1$. The key to this is the realization that (45) can also be rewritten to give bounds on the ratio $V_{0}(x) / V_{-1}(x)=x V_{0}(x)$. We now let

$$
G_{k}^{m}(y)=\frac{k y}{(k-1) y-m+\sqrt{(y+m)^{2}+k y}}
$$

and note that $x g_{k}(x)=G_{k}^{0}\left(x^{2}\right)$ so that (45) is equivalent to

$$
G_{\pi}^{0}\left(x^{2}\right) \leq x V_{0}(x)=\frac{V_{0}(x)}{V_{-1}(x)}<G_{4}^{0}\left(x^{2}\right) .
$$

For integer $m>0$, convexity of $\frac{1}{V_{m}(x)}$ can be shown to be equivalent to

$$
R_{m}(x) \equiv \frac{V_{m}(x)}{V_{m-1}(x)}<G_{4}^{m}\left(x^{2}\right)
$$

In addition to this upper bound, we can show the following

Theorem 21 Let $m \in \mathbf{N}, m \geq 0$. Then the inequalities

$$
G_{8}^{m-1}\left(x^{2}\right)<R_{m}(x)<G_{4}^{m}\left(x^{2}\right)
$$

hold and are optimal in $k$ for the class of functions of the form $G_{k}^{m}\left(x^{2}\right)$.

The upper bound is optimal in $k$ for all $m$. The lower bound is optimal in the sense that 8 is the largest integer for which the lower bound in (50) holds for all $m$. However, as we discuss at the end of Section 5.3, for fixed $m$ one can find $k(m)$ such that $G_{k(m)}^{m-1}\left(x^{2}\right)<R_{m}(x)$ holds with $k(m)>8$.

Since $G_{k}^{m}(y)$ is increasing in both $m$ and $k$, its behavior at zero and infinity allows us to also draw some conclusions about the optimality in $m$ of (50). $R_{m}(0)=$ $1-\frac{1}{2 m}$ and $G_{k}^{m}(0)=1-\frac{1}{1+2 m}$ for all $k$. Therefore, $G_{k}^{\nu}(0)<R_{m}(0)<G_{k^{\prime}}^{\mu}(0)$ implies $\nu \leq m-\frac{1}{2}$ and $\mu \geq m-\frac{1}{2}$ for all $k, k^{\prime}$. Thus, if we insist that $m$ be integer, there 
is no choice of $k$ which allows $m-1$ to be replaced by $m$ in the lower bound when $m>0$, or $m$ by $m-1$ in the upper bound. This argument does not, however, rule out the possibility of bounds of the form $G_{k}^{m-\frac{1}{2}}\left(x^{2}\right)<R_{m}(x)<G_{k^{\prime}}^{m-\frac{1}{2}}\left(x^{2}\right)$.

To examine the behavior at infinity, note that

$$
\begin{aligned}
G_{k}^{m}(y) & =1-\frac{1}{2 y}+\frac{4 m+k+2}{8 y^{2}}+O\left(\frac{1}{y^{3}}\right), \quad \text { and } \\
R_{m}(\sqrt{y}) & =1-\frac{1}{2 y}+\frac{4 m+6}{8 y^{2}}+O\left(\frac{1}{y^{3}}\right)
\end{aligned}
$$

where the asymptotic expansion for $R_{m}$ follows from Proposition 7 . It then follows that $R_{m}(\sqrt{y})<G_{k^{\prime}}^{\mu}(y)$ implies $\mu>m+1-\frac{k}{4}$. Thus $m$ is optimal for the upper bound if $k \leq 4$ and any attempt to decrease $m$ would require an increase in $k$. Furthermore, $\mu=m$ implies $k \geq 4$ so that the upper bound in (50) is optimal in $k$.

We postpone the proof of Theorem 21, which requires a lengthy computation even for the case $p=2$, to the next section. Our proof uses induction on $m$. Therefore, we are able to establish (50) and the theorems in the next section only for $m$ a positive integer. We believe that they are also true for non-integer $m$. However, a proof would require either a different method or independent verification of the upper bound for an initial range, such as $-1<m<0$.

The ratio $R_{m}(x)$ is of interest in its own right, and our results are sufficient to establish that it is increasing in $x$ on $(0, \infty)$. This is proved in the next section after Theorem 23, which uses a similar argument.

Theorem 22 For $m \in \mathbf{N}$, the ratio $R_{m+1}(x)=\frac{V_{m+1}(x)}{V_{m}(x)}$ is increasing in $x$.

\subsection{Convexity of $1 / V_{m}$}

We now prove some important consequences of Theorem 21. The first is

Theorem 23 For all $m \in \mathbf{N}$, the function $1 / V_{m}(x)$ is convex on $[0, \infty)$.

Proof: We need to show that

$$
\left(\frac{1}{V_{m}(x)}\right)^{\prime \prime}=\frac{2\left[V_{m}(x)^{\prime}\right]^{2}-V_{m}(x)\left(V_{m}(x)\right)^{\prime \prime}}{V_{m}(x)^{3}}>0 .
$$

It follows from the differential equation (15) and the recursion relation (24) that

$$
V_{m}(x)^{\prime \prime}=2\left[V_{m}(x)\left(1+2 m+2 x^{2}\right)-2 V_{m-1}(x)\left(x^{2}+m\right)\right]
$$


so that (51) holds if and only if

$$
\left[V_{m}(x)\right]^{2}\left(1+2 m-2 x^{2}\right)+2 V_{m-1}(x) V_{m}(x)\left(3 x^{2}-m\right)-4 x^{2}\left[V_{m-1}(x)\right]^{2} \leq 0 .
$$

After division by $\left[V_{m-1}(x)\right]^{2}$ this can be rewritten as $P\left[R_{m}(x)\right] \leq 0$ where

$$
P(z)=z^{2}\left(1+2 m-2 x^{2}\right)+2 z\left(3 x^{2}-m\right)-4 x^{2} .
$$

Writing the roots of $P(z)=A z+2 B z+C$ in the non-standard form $\frac{-C}{B \pm \sqrt{B^{2}-A C}}$, we find that $G_{4}^{m}\left(x^{2}\right)$ is either the smaller of two positive roots (when $x^{2}>m+\frac{1}{2}$ ) or the only positive root (when $x^{2}<m+\frac{1}{2}$ ). Since $P(0)<0$, in both cases, we can conclude that

$$
z<G_{4}^{m}\left(x^{2}\right) \text { implies } P(z)<0 \text {. }
$$

Therefore, it follows from the upper bound in Theorem 21 that $P\left[R_{m}(x)\right]<0$, hence (51) holds. QED

Proof of Theorem 22: Using (15) one finds that

$$
\frac{d}{d x} R_{m+1}(x)=2 x\left[\frac{R_{m+1}(x)}{R_{m}(x)}-1\right] .
$$

After rewriting this in terms of $V_{m}$ and then using the recursion relation (24) with $p=2$ to eliminate $V_{m+1}$, one finds that $R_{m+1}^{\prime}(x) \geq 0$ if and only if

$$
2(m+1)\left[R_{m}(x)\right]^{2}-\left(2 m+1-2 x^{2}\right) R_{m}(x)-2 x^{2} \leq 0 .
$$

The polynomial $P(z)=2(m+1) z^{2}-\left(2 m+1-2 x^{2}\right) z-2 x^{2}$ has one positive and one negative root, and $R_{m+1}^{\prime}(x) \geq 0$ if and only if $R_{m}(x)$ lies between these two roots. Since $1 \geq R_{m}(x)>0$, it follows that $R_{m+1}(x)$ is increasing if and only if $R_{m}(x)$ is less than the larger root, i.e.,

$$
R_{m}(x) \leq \frac{4 x^{2}}{\sqrt{4\left(x^{2}+m\right)^{2}+1+4 m+12 x^{2}}+2 x^{2}-2 m-1} .
$$

where, we have again written the root in the non-standard form $\frac{C}{-B+\sqrt{B^{2}-A C}}$. Then using the upper bound of Theorem 21, we see that it suffices to show that

$$
\begin{aligned}
R_{m}(x) & \leq \frac{4 x^{2}}{\sqrt{\left(x^{2}+m\right)^{2}+4 x^{2}}+3 x^{2}-m} \\
& \leq \frac{4 x^{2}}{\sqrt{4\left(x^{2}+m\right)^{2}+1+4 m+12 x^{2}}+2 x^{2}-2 m-1}
\end{aligned}
$$


or, equivalently that

$$
\begin{aligned}
\sqrt{\left(x^{2}+m\right)^{2}+4 x^{2}}+3 x^{2}-m \\
\quad \geq \sqrt{4\left(x^{2}+m\right)^{2}+1+4 m+12 x^{2}}+2 x^{2}-2 m-1
\end{aligned}
$$

which is easily checked.

\section{Proof of Ratio Bounds}

The proofs in this section, although elementary, are quite long and tedious. The details were checked using Mathematica.

\subsection{Differential inequality}

In order to prove Theorem 21, it suffices to establish the following

Lemma 24 Let $G_{k}^{m}$ be given by (49). Then

(i) For $m \geq 1, \quad \frac{d}{d x} G_{4}^{m}\left(x^{2}\right) \leq 2 x\left(\frac{G_{4}^{m}\left(x^{2}\right)}{G_{4}^{m-1}\left(x^{2}\right)}-1\right)$.

(ii) For $m \geq 4, \quad \frac{d}{d x} G_{8}^{m}\left(x^{2}\right) \geq 2 x\left(\frac{G_{8}^{m}\left(x^{2}\right)}{G_{8}^{m-1}\left(x^{2}\right)}-1\right)$,

but the inequality (ii) does not hold for $m<4$.

Proof: The proof is based on the elementary principle that if a function on the half-line is zero at the origin and increasing, then it is non-negative. Unfortunately, the actual verification is rather tedious and requires the repeated use of this principle. For simplicity, we put $x^{2}=y$ and assume $y \geq 0$. Then (i) is equivalent to

$$
E_{m}(y) \equiv\left(\frac{G_{4}^{m}(y)}{G_{4}^{m-1}(y)}-1\right)-\frac{d}{d y} G_{4}^{m}(y) \geq 0 .
$$

Let $B_{m}=\left(m^{2}+y^{2}+4 y+2 m y\right)^{\frac{1}{2}}$. Then

$$
G_{4}^{m}(y)=\frac{4 y}{B_{m}+3 y-m} .
$$


and

$$
E_{m}(y)=\frac{B_{m}\left[4 m+\left(B_{m}+3 y-m\right)\left(B_{m-1}-B_{m}+1\right)\right]-\left(4 m^{2}+8 y+4 m y\right)}{B_{m}\left(B_{m}+3 y-m\right)^{2}} .
$$

Thus $E_{m}(y) \geq 0$ if and only if

$$
\begin{aligned}
& B_{m}\left(3 m+B_{m-1}\left[B_{m}+3 y-m\right]\right)+m^{3}+2 m y \\
& \quad \geq 3 m^{2}+4 y+11 y^{2}+m^{2} y+5 m y^{2}+3 y^{3}+B_{m}\left((y+m)^{2}+y\right) .
\end{aligned}
$$

We put

$$
\begin{aligned}
s & =s(y, m)=3 m^{2}+4 y+11 y^{2}+m^{2} y+5 m y^{2}+3 y^{3}, \\
t & =t(y, m)=2 m y+m^{3}, \text { and } \\
h & =h(y, m)=(y+m)^{2}+y-3 m .
\end{aligned}
$$

Then $E_{m}(y) \geq 0$ if and only if

$$
B_{m} B_{m-1}\left(B_{m}+3 y-m\right) \geq B_{m} h+s-t .
$$

Notice that both sides of (53) are positive. For the left side this follows immediately from $B_{m}>m$. For the right, note that $B_{m} h(0)+s(0)-t(0)=0$ and

$$
\begin{aligned}
& \frac{d}{d y}\left[B_{m} h(y)+s(y) t(y)\right] \\
& =\frac{1}{B_{m}}\left[6 y+12 m y+9 m^{2} y+12 y^{2}+9 m y^{2}+3 y^{3}\right. \\
& \left.\quad+B_{m}\left(22 y+10 m y+9 y^{2}\right)+4 B_{m}-2 m B_{m}+3 m^{3}-6 m+m^{2} B_{m}\right] .
\end{aligned}
$$

Now observe that

$$
\begin{aligned}
& 4 B_{m}-2 m B_{m}+3 m^{3}-6 m+m^{2} B_{m} \\
& \quad=3 m\left(m^{2}-2\right)+B_{m}\left(m^{2}-2 m+4\right) \geq 3 m\left(m^{2}-1\right) \geq 0 .
\end{aligned}
$$

since $m \geq 1$ and $B_{m} \geq m$. Hence $B_{m} h+s-t$ is increasing in $y$ and the right side of (53) is also positive. Therefore we can square both sides of (53) to conclude that it is equivalent to

$$
F(y)=B_{m} f_{1}(y)-f_{2}(y) \geq 0
$$


where

$$
f_{1}(y)=(m+y)\left(y^{3}+m y^{2}+3 y^{2}-m^{2} y-3 m y+2 y-m^{3}+2 m^{2}\right)
$$

and

$$
\begin{aligned}
f_{2}(y)=y^{5} & +3 m y^{4}+5 y^{4}+2 m^{2} y^{3}+5 m y^{3}+2 y^{3} \\
& -2 m^{3} y^{2}-3 m^{2} y^{2}-3 m^{4} y-m^{3} y+6 m^{2} y-m^{5}+2 m^{4} .
\end{aligned}
$$

Note that $F(0)=0$. Therefore, to prove (54) it is enough to show that $\frac{d}{d y} F(y)=$ $B_{m} f_{1}^{\prime}(y)+\frac{f_{1}(y)(2+y+m)}{B_{m}}-f_{2}^{\prime}(y) \geq 0$, or equivalently

$$
D(y) \equiv d_{1}(y)-B_{m} d_{2}(y) \geq 0
$$

where

$$
\begin{aligned}
d_{1}(y)= & B_{m}^{2} f_{1}^{\prime}(y)+f_{1}(y)(2+y+m) \\
= & 6 m^{3}-m^{4}-3 m^{5}+12 m y+4 m^{2} y-13 m^{3} y-7 m^{4} y+20 y^{2} \\
& +14 m y^{2}+7 m^{2} y^{2}+2 m^{3} y^{2}+48 y^{3}+49 m y^{3}+18 m^{2} y^{3}+30 y^{4} \\
& +17 m y^{4}+5 y^{5}, \text { and } \\
d_{2}(y)= & f_{2}^{\prime}(y)=6 m^{2}-m^{3}-3 m^{4}-6 m^{2} y-4 m^{3} y \\
& \quad+6 y^{2}+15 m y^{2}+6 m^{2} y^{2}+20 y^{3}+12 m y^{3}+5 y^{4} .
\end{aligned}
$$

Note that $D(0)=0$. Therefore, to prove (55) it is enough to show that $\frac{d}{d y} D(y)=$ $d_{1}^{\prime}(y)-B_{m} d_{2}^{\prime}(y)-\frac{d_{2}(y)(2+y+m)}{B_{m}} \geq 0$, or equivalently,

$$
G(y) \equiv B_{m} g_{1}(y)-g_{2}(y) \geq 0
$$

where

$$
\begin{aligned}
g_{1}(y)= & d_{1}^{\prime}(y) \\
= & 12 m+4 m^{2}-13 m^{3}-7 m^{4}+40 y+28 m y+14 m^{2} y+4 m^{3} y \\
& \quad+144 y^{2}+147 m y^{2}+54 m^{2} y^{2}+120 y^{3}+68 m y^{3}+25 y^{4}, \text { and } \\
g_{2}(y)= & B_{m}^{2} d_{2}^{\prime}(y)+d_{2}(y)(2+y+m) \\
= & 12 m^{2}+4 m^{3}-13 m^{4}-7 m^{5}-18 m^{2} y-13 m^{3} y-3 m^{4} y \\
& +60 y^{2}+180 m y^{2}+183 m^{2} y^{2}+58 m^{3} y^{2}+298 y^{3} \\
& +353 m y^{3}+122 m^{2} y^{3}+170 y^{4}+93 m y^{4}+25 y^{5}
\end{aligned}
$$


Note that $G(0)=0$. Therefore, to prove (56) it is enough to show that $\frac{d}{d y} G(y)=$ $B_{m} g_{1}^{\prime}(y)+\frac{g_{1}(y)(2+y+m)}{B_{m}}-g_{2}^{\prime}(y) \geq 0$, or equivalently

$$
H(y)=h_{1}(y)-B_{m} h_{2}(y) \geq 0,
$$

where

$$
\begin{aligned}
h_{1}(y)= & B_{m}^{2} g_{1}^{\prime}(y)+g_{1}(y)(2+y+m) \\
= & 24 m+60 m^{2}+6 m^{3}-13 m^{4}-3 m^{5}+240 y+300 m y+460 m^{2} y \\
& \quad+347 m^{3} y+113 m^{4} y+1520 y^{2}+2246 m y^{2}+1663 m^{2} y^{2}+482 m^{3} y^{2} \\
& \quad+2112 y^{3}+2233 m y^{3}+738 m^{2} y^{3}+930 y^{4}+497 m y^{4}+125 y^{5}, \quad \text { and } \\
h_{2}(y)= & g_{2}^{\prime}(y) \\
= & -18 m^{2}-13 m^{3}-3 m^{4}+120 y+360 m y+366 m^{2} y+116 m^{3} y \\
& +894 y^{2}+1059 m y^{2}+366 m^{2} y^{2}+680 y^{3}+372 m y^{3}+125 y^{4} .
\end{aligned}
$$

Note that $H(0)=12 m\left(2+5 m+2 m^{2}\right)>0$. Therefore, to prove (57) it is enough to show that $\frac{d}{d y} H(y)=h_{1}^{\prime}(y)-B_{m} h_{2}^{\prime}(y)-\frac{h_{2}(y)(2+y+m)}{B_{m}} \geq 0$, or equivalently

$$
l_{1}(y) B_{m}-l_{2}(y) \geq 0
$$

where

$$
\begin{aligned}
l_{1}(y)= & h_{1}^{\prime}(y) \\
= & 240+300 m+460 m^{2}+347 m^{3}+113 m^{4}+3040 y+4492 m y \\
& +3326 m^{2} y+964 m^{3} y+6336 y^{2}+6699 m y^{2}+2214 m^{2} y^{2}+ \\
& +3720 y^{3}+1988 m y^{3}+625 y^{4}, \quad \text { and } \\
l_{2}(y)= & B_{m}^{2} h_{2}^{\prime}(y)+h_{2}(y)(2+y+m) \\
= & 84 m^{2}+316 m^{3}+347 m^{4}+113 m^{5}+720 y+2520 m y+5046 m^{2} y \\
& +3899 m^{3} y+1077 m^{4} y+9180 y^{2}+15780 m y^{2}+11727 m^{2} y^{2} \\
& +3178 m^{3} y^{2}+12202 y^{3}+13145 m y^{3}+4202 m^{2} y^{3}+4970 y^{4} \\
& +2613 m y^{4}+625 y^{5} .
\end{aligned}
$$

Note that $l_{1}(y) \geq 0$ and $l_{2}(y) \geq 0$ for all $y \geq 0$. Therefore (58) holds, if and only if $L(y)=B_{m}^{2}\left(l_{1}(y)\right)^{2}-\left(l_{2}(y)\right)^{2} \geq 0$, which follows immediately from the fact that all the coefficients are positive in

$$
\begin{aligned}
L(y)= & 4\left(14400 m^{2}+36000 m^{3}+75936 m^{4}+97368 m^{5}+78972 m^{6}+37188 m^{7}\right. \\
& +8136 m^{8}+57600 y+172800 m y+717360 m^{2} y+1373400 m^{3} y
\end{aligned}
$$




$$
\begin{aligned}
& +1732428 m^{4} y+1360314 m^{5} y+599454 m^{6} y+111444 m^{7} y+1344000 y^{2} \\
& +3838560 m y^{2}+8437260 m^{2} y^{2}+11062920 m^{3} y^{2}+8495031 m^{4} y^{2} \\
& +3499083 m^{5} y^{2}+595986 m^{6} y^{2}+9342880 y^{3}+24217360 m y^{3} \\
& +32546720 m^{2} y^{3}+24561680 m^{3} y^{3}+9950080 m^{4} y^{3}+1694280 m^{5} y^{3} \\
& +17918380 y^{4}+37038224 m y^{4}+34271234 m^{2} y^{4}+15627870 m^{3} y^{4} \\
& +2862630 m^{4} y^{4}+15343236 y^{5}+23700982 m y^{5}+13930330 m^{2} y^{5} \\
& +2982516 m^{3} y^{5}+6445963 y^{6}+6618363 m y^{6}+1887294 m^{2} y^{6} \\
& \left.+1302640 y^{7}+667056 m y^{7}+101250 y^{8}\right) .
\end{aligned}
$$

To prove (ii) we proceed similarly, but now let $B_{m}(y)=\sqrt{(y+m)^{2}+8 y}$ and $E_{m}(y)=\frac{d}{d y} G_{8}^{m}(y)-\left(\frac{G_{8}^{m}(y)}{G_{8}^{m-1}(y)}-1\right)$, noting that

$$
G_{8}^{m}(y)=\frac{8 y}{B_{m}+7 y-m} .
$$

We now need to show that $E_{m}(y) \geq 0$ for all $m \geq 4$. As the argument is similar to that above, we omit the details except to indicate the steps leading to the condition $m \geq 4$. Observe that $E_{m}(y) \geq 0$ if and only if

$$
\begin{gathered}
B_{m}\left((y+m)^{2}+y-7 m\right) \\
+7 m^{2}-m^{3}+24 y-2 m y+5 m^{2} y+55 y^{2}+13 m y^{2}+7 y^{3} \\
\geq B_{m} B_{m-1}\left(7 y-m+B_{m}\right) .
\end{gathered}
$$

Again, both sides of the inequality are positive. Hence we can square both sides of the inequality and, as above get that (59) is equivalent to

$$
F(y)=f_{1}(y)-B_{m} f_{2}(y) \geq 0,
$$

with the appropriate $f_{1}$ and $f_{2}$. Again $F(0)=0$. Therefore, in order to prove (60), it is enough to show that $\frac{d}{d y} F(y) \geq 0$, or equivalently, after rewriting,

$$
D(y)=B_{m} d_{1}(y)-d_{2}(y) \geq 0,
$$

with the appropriate $d_{1}$ and $d_{2}$. And again $D(0)=0$. We repeat the procedure: To prove (61), it is enough to show that $\frac{d}{d y} D(y) \geq 0$, or equivalently,

$$
e_{1}(y)-B_{m} e_{2}(y) \geq 0,
$$

with the appropriate $e_{1}$ and $e_{2}$. $e_{1}$ and $e_{2}$ turn out to be both positive for $y \geq 0$. Therefore (61) holds if

$$
L(y)=\left(e_{1}(y)\right)^{2}-\left(B_{m} e_{2}(y)\right)^{2} \geq 0 .
$$


$L(0)=0$ and

$$
L^{\prime}(0)=192 m^{2}(m-4)(1+2 m)\left(480+64 m+90 m^{2}+33 m^{3}\right) .
$$

Thus $L^{\prime}(0) \geq 0$ if and only if $m \geq 4$. For all $m \geq 4, L^{\prime \prime}(y) \geq 0$ for all $y \geq 0$. This finishes (ii). QED

\subsection{Proof of Theorem 21}

We will prove by induction that $R_{m}(x)<G_{4}^{m}\left(x^{2}\right)$ for $m=0,1,2,3, \ldots$ As observed earlier, this inequality holds for $m=0$, since it is then equivalent to the upper bound in (45). Let

$$
H_{m}(x)=G_{4}^{m}\left(x^{2}\right)-R_{m}(x) .
$$

Then the upper bound in Theorem 21 is equivalent to $H_{m}(x) \geq 0$. This can be verified using the strategy of Section 4.1 if the following conditions hold

(i) $H_{m}(0)>0$.

(ii) $\lim _{x \rightarrow \infty} H_{m}(x)=0$.

(iii) $H_{m}^{\prime}(x) \leq F_{\mathrm{pos}}(x) H_{m}(x)$ for some strictly positive function $F_{\mathrm{pos}}(x)>0$.

Conditions (i) and (ii) hold. Indeed,

$$
H_{m}(0)=\frac{2 m}{1+2 m}-\frac{\Gamma(m) \Gamma\left(m+\frac{1}{2}\right)}{\Gamma(m+1) \Gamma\left(m-\frac{1}{2}\right)}=\frac{1}{2 m(1+2 m)},
$$

and

$$
\lim _{x \rightarrow \infty} H_{m}(x)=0,
$$

since $\lim _{x \rightarrow \infty} R_{m}(x)=1$, and for all $k \geq 1, \lim _{x \rightarrow \infty} G_{k}^{m}(x)=1$.

We check now condition (iii). It follows from Lemma 24 (i) and (52) that

$$
\begin{aligned}
H_{m}^{\prime}(x) & \leq 2 x\left[\frac{G_{4}^{m}\left(x^{2}\right)}{G_{4}^{m-1}\left(x^{2}\right)}-\frac{R_{m}(x)}{R_{m-1}(x)}\right] \\
& =\frac{2 x}{G_{4}^{m-1}\left(x^{2}\right) R_{m-1}(x)}\left[G_{4}^{m}\left(x^{2}\right) R_{m-1}(x)-G_{4}^{m-1}\left(x^{2}\right) R_{m}(x)\right] \\
& \leq \frac{2 x}{R^{m-1}(x)}\left[G_{4}^{m}\left(x^{2}\right)-R_{m}(x)\right] \\
& =\frac{2 x}{R^{m-1}(x)} H_{m}(x)
\end{aligned}
$$


where the inequality follows from the induction hypothesis $R_{m-1}(x)<G_{4}^{m-1}\left(x^{2}\right)$. Thus (iii) holds with $F_{\text {pos }}(x)=2 x / R_{m-1}(x)$.

For $m \geq 4$, the lower bound is proved similarly. One considers $H_{m}(x)=$ $R_{m}(x)-G_{8}^{m}\left(x^{2}\right)$ instead and uses Lemma 24(ii). The cases $R_{1}, R_{2}$ and $R_{3}$ have to be verified directly as Lemma 24 (ii) only covers the cases $R_{m}$ for $m \geq 4$.

Using (24), $R_{1} \geq G_{8}^{0}$ is equivalent to showing that

$$
\frac{x}{V_{0}(x)} \geq \frac{9 x+14 x^{3}+\left(2 x^{2}-1\right) \sqrt{8+x^{2}}}{2\left(7 x+\sqrt{8+x^{2}}\right)} .
$$

As $V_{0}$ is always positive, this inequality holds trivially for those $x$, for which the right hand side is negative or zero. Therefore we only need to prove the inequality on the interval $\left[x_{0}, \infty\right), x_{0} \simeq 0.2511$, where

$$
9 x+14 x^{3}+\left(2 x^{2}-1\right) \sqrt{8+x^{2}} \geq 0 .
$$

Hence we need to show that for all $x \in\left[x_{0}, \infty\right)$,

$$
\begin{aligned}
V_{0}(x) & \leq 2 x \frac{7 x+\sqrt{8+x^{2}}}{9 x+14 x^{3}+\left(2 x^{2}-1\right) \sqrt{8+x^{2}}} \\
& =2 x \frac{6 x^{2}-1}{1+6 x^{2}+12 x^{4}-2 x \sqrt{8+x^{2}}} .
\end{aligned}
$$

Put $h_{1}(x)=2 x \frac{6 x^{2}-1}{1+6 x^{2}+12 x^{4}-2 x \sqrt{8+x^{2}}}$. By Theorem 20 of Section 4.1, inequality 62) is true for all $x \in\left[x_{0}, \infty\right)$, for which

$$
g_{4}(x) \leq h_{1}(x)
$$

This last inequality holds only on an interval $\left[x_{0}, x_{1}\right], x_{1} \simeq 1.399$. For all $x \geq x_{1}$, we show that

$$
h_{1}(x)<\frac{1}{x}
$$

and

$$
h_{1}^{\prime} \leq 2\left(x h_{1}-1\right)
$$

Then (62) follows as in Section 4.1.

Next, we find that $R_{2} \geq G_{8}^{1}$ is equivalent to $V_{0}(x) \geq h_{2}(x)$, where

$$
h_{2}(x)=2 x \frac{\left.3+9 x^{2}+14 x^{4}+\left(2 x^{2}-3\right)\left(8 x^{2}+\left(1+x^{2}\right)^{2}\right)\right)^{\frac{1}{2}}}{\left.-3-7 x^{2}+32 x^{4}+28 x^{6}+\left(3-4 x^{2}+4 x^{4}\right)\left(8 x^{2}+\left(1+x^{2}\right)^{2}\right)\right)^{\frac{1}{2}}}
$$


and $R_{3} \geq G_{8}^{2}$ is equivalent to $V_{0}(x) \leq h_{3}(x)$ where

$h_{3}(x)=\frac{2 x}{N(x)}\left[-30-23 x^{2}+32 x^{4}+28 x^{6}+\sqrt{8 x^{2}+\left(2+x^{2}\right)^{2}}\left(15-8 x^{2}+4 x^{4}\right)\right]$,

with

$$
\begin{aligned}
& N(x)=30+3 x^{2}-42 x^{4}+92 x^{6}+56 x^{8} \\
& \left.\quad+\left(8 x^{2}+\left(2+x^{2}\right)^{2}\right)\right)^{\frac{1}{2}}\left(-15+18 x^{2}-12 x^{4}+8 x^{6}\right) .
\end{aligned}
$$

Again, we have to check these inequalities for $V_{0}$ only for those $x$, for which the right hand sides are positive. We then proceed as for $R_{1}$ and show that $g_{\pi} \geq h_{2}$ up to a certain $x_{2}$ and that $h_{2}<\frac{1}{x}, h_{2}^{\prime} \geq 2\left(x h_{1}-1\right)$ on $\left[x_{2}, \infty\right)$. Similarly, we show that $g_{4} \leq h_{3}$ up to a certain $x_{3}$ and that $h_{3}<\frac{1}{x}, h_{3}^{\prime} \leq 2\left(x h_{1}-1\right)$ on $\left[x_{3}, \infty\right)$. QED

Note that these arguments also show that on the interval $\left[x_{1}, \infty\right)$ the function $h_{1}$ is a better upper bound for $V_{0}$ than $g_{4}$; on $\left[x_{2}, \infty\right)$ the function $h_{2}$ is a better lower bound for $V_{0}$ than $g_{\pi}$; and on $\left[x_{3}, \infty\right)$ the function $h_{3}$ is a better upper bound for $V_{0}$ than $g_{4}$. In fact, $h_{3} \leq h_{1} \leq g_{4}$ for $x>x_{3}$.

\subsection{Optimality of bounds}

We still need to consider optimality of the lower bound in upper bound in 50 in the parameter $k$. We continue the strategy above using similar notation so that now $B_{m}=\sqrt{(y+m)^{2}+k y}$ and $E_{m}(y)=\left[\frac{G_{k}^{m}(y)}{G_{k}^{m-1}(y)}-1\right]-\frac{d}{d y} G_{k}^{m}(y)$ with

$$
G_{k}^{m}(y)=\frac{k y}{B_{m}+(k-1) y-m} .
$$

Then $E_{m}(y) \leq 0$ if and only if,

$$
2 B_{m} B_{m-1}\left(B_{m}+(k-1) y-m\right) \leq 2 B_{m}\left(y+(y+m)^{2}-(k-1) m\right)+P
$$

where

$$
\begin{aligned}
P=-2 m^{2} & +2 k m^{2}-2 m^{3}-2 k y+k^{2} y-4 m y-6 m^{2} y+2 k m^{2} y \\
& -2 y^{2}-2 k y^{2}+2 k^{2} y^{2}-6 m y^{2}+4 k m y^{2}-2 y^{3}+2 k y^{3} .
\end{aligned}
$$

For $m \geq 1$ and $k \geq 2$ both sides of the inequality are positive. Therefore we can square both sides and get that $E_{m}(y) \leq 0$ if and only if

$$
F(y)=f_{1}(y)-B_{m} f_{2}(y) \geq 0
$$


where

$$
\begin{aligned}
f_{1}(y)= & -16 k m^{4}+8 k^{2} m^{4}+32 m^{5}-16 k m^{5}-20 k^{2} m^{2} y+8 k^{3} m^{2} y+8 k m^{3} y \\
& -4 k^{2} m^{3} y+160 m^{4} y-96 k m^{4} y+8 k^{2} m^{4} y-4 k^{3} y^{2}+k^{4} y^{2} \\
& +120 k m^{2} y^{2}-84 k^{2} m^{2} y^{2}+12 k^{3} m^{2} y^{2}+320 m^{3} y^{2}-224 k m^{3} y^{2} \\
& +32 k^{2} m^{3} y^{2}+20 k^{2} y^{3}-20 k^{3} y^{3}+4 k^{4} y^{3}+152 k m y^{3}-124 k^{2} m y^{3} \\
& +24 k^{3} m y^{3}+320 m^{2} y^{3}-256 k m^{2} y^{3}+48 k^{2} m^{2} y^{3}+56 k y^{4}-52 k^{2} y^{4} \\
& +12 k^{3} y^{4}+160 m y^{4}-144 k m y^{4}+32 k^{2} m y^{4}+32 y^{5}-32 k y^{5}+8 k^{2} y^{5}, \\
f_{2}(y)= & 4\left(-4 k m^{3}+2 k^{2} m^{3}+8 m^{4}-4 k m^{4}-3 k^{2} m y+k^{3} m y+2 k m^{2} y\right. \\
& -k^{2} m^{2} y+32 m^{3} y-20 k m^{3} y+2 k^{2} m^{3} y+k^{2} y^{2}+16 k m y^{2}-12 k^{2} m y^{2} \\
& +2 k^{3} m y^{2}+48 m^{2} y^{2}-36 k m^{2} y^{2}+6 k^{2} m^{2} y^{2}+10 k y^{3}-9 k^{2} y^{3}+2 k^{3} y^{3} \\
& \left.+32 m y^{3}-28 k m y^{3}+6 k^{2} m y^{3}+8 y^{4}-8 k y^{4}+2 k^{2} y^{4}\right) .
\end{aligned}
$$

Then $F(0)=0$ and in order that $F \geq 0$, we must have $\frac{d}{d y} F(0) \geq 0$. Computing $\frac{d}{d y} F(y)$, we find that $\frac{d}{d y} F(0)=0$. We apply the same procedure as in the proof of Lemma 24, compute the successive derivatives and evaluate them at 0. Evaluating the derivative at 0 , in the fourth step of the procedure gives the value

$$
24 k^{3} m(1+2 m)(k m-6 m-k) \text {. }
$$

Therefore, in order that (63) (which is the condition for the lower bound) holds for all $m \geq 2$, we have to have at least that $k \geq \lim _{m \rightarrow \infty} \frac{6 m}{m-1}=6$. Thus for $m=2, k \geq 12$ will do, for $m=3, k \geq 9$, for $m=4, k \geq 8$ and so fourth. Therefore, as $G_{k}^{m}$ is increasing in $k$, it seems a natural choice to pick $k=12$ or bigger for the lower bound. And indeed, one can check that $G_{k}^{m}$ satisfies the lower bound condition of Lemma 24 for $k \geq 12$ and $m \geq 2$. However, it is not true that for $k>8, G_{k}^{m-1}$ is a lower bound for $R_{m}$, for all $m \geq 1$. It is a lower bound for all $m \geq m(k)$, from a certain $m(k)$ on. Thus the induction in the proof of Theorem 21 cannot start at $m=0$ or $m=1$. For $m<m(k)$, there exists $x_{m}$ such that $R_{m}-G_{k}^{m-1} \geq 0$ on $\left[0, x_{m}\right]$ and $R_{m}-G_{k}^{m-1}<0$ on $\left(x_{m}, \infty\right)$.

\subsection{Extensions to general $p$}

For $p=1$, all the functions involved are identically equal to 1 and hence trivially convex. For large $x, 1 / V_{m}^{p}(x) \approx x^{p-1}$ and $x^{p-1}$ is concave for $1<p<2$. Hence we can not expect convexity of $1 / V_{m}^{p}$ on $(0, \infty)$ for $p$ in $(1,2)$. It was shown in [12] that $\frac{1}{V_{0}^{p}}$ is not convex on $\mathbf{R}^{+}$for $0<p<1$. Therefore, we study only generalizations to $p>2$. Our method of proof yields verification of the convexity 
of $\frac{1}{V_{m}^{p}}$ for all $m \geq 1$ up to at least $p=4$. However this method breaks down for larger $p$.

We generalize our previous notation to $R_{m}^{p}(x)=\frac{V_{m}^{p}(x)}{V_{m-1}^{p}(x)}$, and observe that

$$
\frac{d}{d x} R_{m}^{p}(x)=p x^{p-1}\left[\frac{R_{m}^{p}(x)}{R_{m-1}^{p}(x)}-1\right] .
$$

For $k \geq 1, m \geq 0, p \geq 1$ we generalize $G_{k}^{m}$ to

$$
G_{k}^{m, p}\left(x^{p}\right)=\frac{k p x^{p}}{p\left[(k-1) x^{p}-m\right]+\sqrt{p^{2}\left(x^{p}+m\right)^{2}+2 k p(p-1) x^{p}}} .
$$

The proofs of Theorems 21, 22 and 23 can be extended provided that the analogue of Lemma 24 holds. This is not the case for large $p$. However, although the generalization of the upper bound in Theorem 21 is a necessary and sufficient condition for convexity of $1 / V_{m}^{p}(x)$, Lemma 24 is only a sufficient condition for Theorem 21. Indeed, we were able to establish the lower bound in Theorem 21 for $m=1,2,3$ even though part (ii) of Lemma 24 does not hold for $m<4$. Hence, the fact that Lemma 24 breaks down for large $p$ does not preclude convexity of $1 / V_{m}^{p}(x)$. On the contrary, numerical evidence suggests that $\frac{1}{V_{m}^{p}}$ is convex for all $p \geq 2$.

Lemma 25 For all $4 \geq p \geq 2, m \geq 1$,

$$
\frac{d}{d x} G_{4}^{m, p}\left(x^{p}\right) \leq p x^{p-1}\left[\frac{G_{4}^{m, p}\left(x^{p}\right)}{G_{4}^{m-1, p}\left(x^{p}\right)}-1\right]
$$

This is equivalent to

$$
E_{m}^{p}=p x^{p-1}\left[\frac{G_{4}^{m, p}\left(x^{p}\right)}{G_{4}^{m-1, p}\left(x^{p}\right)}-1\right]-\frac{d}{d x} G_{4}^{m, p}\left(x^{p}\right) \geq 0
$$

which allows us to make some remarks about the range of validity. Although Lemma 25 can probably be extended to some higher $p$, it does not hold for all $p, m$. On the contrary, for all $m \geq 1$ there exists $p(m)$ and an interval $\left(x_{1}^{p(m)}, x_{2}^{p(m)}\right)$ such that $E_{m}^{p}<0$ on that interval for all $p \geq p(m)$. For example, numerical results show that for $m=1$, an interval on which $E_{m}<0$ exists when $p \geq 10$; for $m=2$ when $p \geq 14$ and for $m=3$ when $p \geq 18$.

For simplicity, we only sketch the proof of Lemma 25 and give the final expressions for $p=3$. Similar expressions can be given for $p=4$. We have checked the details using Mathematica, but omit the long formulas. As the expressions involved are monotone in $p$, this suffices for the entire interval $2 \leq p \leq 4$. 
Proof: Let $B_{m}=\sqrt{p^{2}(y+m)^{2}+8 p(p-1) y}$. Then $E_{m}^{p} \geq 0$ is equivalent to

$$
\begin{aligned}
& B_{m} B_{m-1}\left(B_{m}+3 p y-p m\right) \\
& \quad \geq \quad p B_{m}\left[p(y+m)^{2}+y(5 p-8)-3 p m\right]+p^{2}\left\{3 m^{2} p-m^{3} p\right. \\
& \left.\quad+y\left(-8+8 m+8 p-6 m p+m^{2} p\right)+y^{2}(-24+23 p+5 m p)+3 p y^{3}\right\} .
\end{aligned}
$$

Following the procedure used to prove Lemma 24 (i), we eventually find that it would suffice to show that

$$
l_{1}(y) B_{m}-l_{2}(y) \geq 0
$$

where, for $p=3$,

$$
\begin{aligned}
l_{1}(y)= & 3\left(5120+2880 m+13800 m^{2}+11034 m^{3}+3051 m^{4}+169600 y\right. \\
& +199632 m y+116820 m^{2} y+26028 m^{3} y+298296 y^{2}+239706 m y^{2} \\
& \left.+59778 m^{2} y^{2}+133920 y^{3}+53676 m y^{3}+16875 y^{4}\right), \quad \text { and } \\
l_{2}(y)= & 27\left(-2048 m+192 m^{2}+3448 m^{3}+3678 m^{4}+1017 m^{5} 25600 y\right. \\
& +49920 m y+76152 m^{2} y+45330 m^{3} y+9693 m^{4} y+200000 y^{2} \\
& +250152 m y^{2}+139266 m^{2} y^{2}+28602 m^{3} y^{2}+195880 y^{3} \\
& \left.+157254 m y^{3}+37818 m^{2} y^{3}+59640 y^{4}+23517 m y^{4}+5625 y^{5}\right) .
\end{aligned}
$$

Both $l_{1}(y)$ and $l_{2}(y)$ in $(65)$ are positive, and hence (65) is equivalent to

$$
L=l_{1}(y)^{2} B_{m}^{2}-l_{2}(y)^{2} \geq 0 .
$$

which can be verified for $m \geq 1$. QED

Acknowledgment: It is a pleasure to thank Professor S. Kwapien for the argument leading to the upper bound in Property (a), and Professors R. Askey and M. Ismail for helpful discussions about the properties of hypergeometric functions. We would also like to thank Professor V. Mascioni for providing a copy of [12] before publication, and Professor Fink for drawing our attention to reference [14].

\section{References}

[1] G.E. Andrews, R. Askey, R. Roy Special Functions (Cambridge University press, 1999). 
[2] Y. Avron, I. Herbst, and B. Simon, "Strongly Bound States of Hydrogen in Intense Magnetic Field" Phys. Rev. A 20, 2287-2296 (1979).

[3] H. Bateman, Higher Transcendental Functions, Volume 1 (McGraw-Hill, 1953).

[4] R. B. Boas, Jr. and R.C. Buck Polynomial Expansions of Analytic Functions (Academic Press, 1964).

[5] A.V. Boyd, "Inequalities for the Mills' Ratio" Rep. Statist. Appl. Res. Un. Jap. Sci. Engrs. 6, 44-46 (1959).

[6] A.V. Boyd, "Note on a Paper by Uppuluri" Pacific J. Math. 22, 9-10 (1967).

[7] R. Brummelhuis and M. B. Ruskai, "A One-Dimensional Model for ManyElectron Atoms in Extremely Strong Magnetic Fields: Maximum Negative Ionization", J. Phys. A, 32, 2567-2582 (1999).

[8] R. Brummelhuis and M. B. Ruskai, "One-Dimensional Models for ManyElectron Atoms in Extremely Strong Magnetic Fields: Anti-symmetry in the Landau Levels", preprint in preparation.

[9] K. Ito and H.P. McKean Diffusion Processes and Their Sample Paths p. 17, (Springer-Verlag, 1965) .

[10] W. Gautschi, "Some Elementary Inequalities Relating to the Gamma and Incomplete Gamma Function" J. Math. and Phys. 38, 77-81 (1959).

[11] N.N. Lebedev, Special Functions and Their Applications, English edition translated and revised by R.A. Silverman, (Prentice-Hall, 1965).

[12] V. Mascioni, "A generalization of an inequality related to the error function", preprint

[13] E.H. Lieb, J.P. Solovej and J. Yngvason, "Heavy Atoms in the Strong Magnetic Field of a Neutron Star" Phys. Rev. Lett. 69, 749-752 (1992); and "Asymptotics of Heavy Atoms in High Magnetic Fields I: Lowest Landau Band Regions", Commun. Pure Appl. Math. 47, 513-591 (1993).

[14] D.S. Mitronović Analytic Inequalities, Section 2.26, (Springer-Verlag, 1970).

[15] M.B. Ruskai and E. Werner, "A Pair of Optimal Inequalities Related to the Error Function", preprint 97-564 on the Texas Mathematical Physics Preprint Archive http://www.ma.utexas.edu/mp_arc/ 
[16] E. Stein, Singular Integrals and Differentiability Properties of Functions (Princeton Univ. Press, 1970)

[17] S. J. Szarek and E. Werner, "Confidence Regions for Means of Multivariate Normal Distributions and a Non-symmetric Correlation Inequality for Gaussian Measure", MSRI preprint No. 1997-009, to appear in J. Multivariate Analysis.

[18] M. Wirth, "On considère la fonction de $\mathbf{R}$ dans $\mathbf{R}$ défine par $f(x)=e^{-x^{2} / 2}$; démontrer que la fonction $g$ de $\mathbf{R}$ dans $\mathbf{R}$ défine par $g(x)=f(x) / \int_{x}^{\infty} f(t) d t$ est convexe", Revue de Mathématiques Spéciales 104, 187-88 (1993).

[19] L. Schiff and H. Snyder, "Theory of the Quadratic Zeeman Effect", Phys. Rev. 55, 59-63 (1939). 